\title{
ON THE ACTION OF THE UNITARY GROUP ON THE PROJECTIVE PLANE OVER A LOCAL FIELD
}

\author{
HARM VOSKUIL
}

(Received 29 January 1996; revised 30 September 1996)

Communicated by R. Howlett

\begin{abstract}
Let $G$ be a unitary group of rank one over a non-archimedean local field $K$ (whose residue field has a characteristic $\neq 2$ ). We consider the action of $G$ on the projective plane. A $G(K)$ equivariant map from the set of points in the projective plane that are semistable for every maximal $K$ split torus in $G$ to the set of convex subsets of the building of $G(K)$ is constructed. This map gives rise to an equivariant map from the set of points that are stable for every maximal $K$ split torus to the building. Using these maps one describes a $G(K)$ invariant pure affinoid covering of the set of stable points. The reduction of the affinoid covering is given.
\end{abstract}

1991 Mathematics subject classification (Amer. Math. Soc.): primary 32P05; secondary 14M17, 20 G25.

\section{Introduction}

Let $K$ be a non-archimedean local field. We assume that the characteristic of the residue field of $K$ is $\neq 2$. For a separable algebraic extension $L \supset K$ of degree two, we consider the action of the unitary group $S U_{3}(L)$ on $\mathbb{P}_{L}^{2}$. The rank of the unitary group is assumed to be one.

Let $Y^{s s}$ and $Y^{s}$ be the subspaces of $\mathbb{P}_{L}^{2}$ consisting of the points that are semistable and stable, respectively, for every maximal $K$-split torus $S \subset S U_{3}(L)$. Here one takes the $S$-linearization coming from the (unique) $S U_{3}(L)$-linearization of some ample line bundle on $\mathbb{P}_{L}^{2}$. All ample line bundles give the same set of (semi-) stable points, since they are all powers of the ample line bundle $\mathscr{O}(1)$.

Let $B$ denote the Bruhat-Tits building of $S U_{3}(L)$. Since the rank of $S U_{3}(L)$ is one, the building is a tree.

This work is supported by the Australian Research Council.

(C) 1997 Australian Mathematical Society 0263-6115/97\$A2.00+0.00 
We construct a map $I: Y^{s s} \longrightarrow$ convex subsets of $B$ \} that is $S U_{3}(L)$-equivariant. A complete description of the convex subsets that are in the image of $I$ is given (See Theorems 5.10 and 6.2). In particular we prove that $I(x)$ is bounded if and only if $x \in Y^{s}$.

The map $I$ is then used to construct a pure affinoid covering of the rigid analytic space $Y^{s}$. The components of the reduction of $Y^{s}$ with respect to this affinoid covering are proper. There is a 1-1 correspondance between these components and certain bounded convex subsets of the building $B$ (See Theorem 7.5). In the last paragraph we describe the components of the reduction of $Y^{s}$. Giving a reduction of $Y^{s}$ is equivalent to giving a formal scheme over $L^{0}$, the ring of integers of $L$, that has as its generic fibre the analytic space $Y^{s}$ and as its closed fibre the reduction of $Y^{s}$.

Let $\Gamma \subset S U_{3}(L)$ be a discrete and co-compact subgroup. Then $Y^{s} / \Gamma$ is a separated rigid analytic space. Since the group $\Gamma$ has infinitely many orbits on the components of the reduction of $Y^{s}$, the quotient is not proper. Moreover we do not expect that the quotient itself can be compactified (See 8.13). This is in contrast with similar spaces considered in [4]. There one always assumes that the sets of stable and semistable points coincide. Then the quotient is proper. On the quotients of the spaces considered in [4] there exist no non-constant meromorphic functions (except for some cases related to Drinfeld's symmetric space). On the quotients $Y^{s} / \Gamma$ of the space $Y^{s}$ studied here there do exist non-constant meromorphic functions. This will be treated in a forthcoming paper.

Finally I would like to thank Marius van der Put and King Lai for their help and encouragement and Michel Gros for asking too many questions about $S_{3}(L)$.

\section{The group $S U_{3}(L)$ and its building}

\subsection{Notation.}

(1) $K$ : a non-archimedean local field with $\operatorname{Char}(\bar{K}) \neq 2$.

(2) $\bar{K}$ : the residue field of $K$.

(3) $L \supset K$ : a separable algebraic extension of $K$ of degree 2 .

(4) $\pi$ : a generator of the maximal ideal of $L^{0}$.

(5) $\tau$ : the generator of $\operatorname{Gal}(L / K)$; we write $\bar{x}:=\tau(x)$ for $x \in L$.

(6) $K^{0}, L^{0}$ : the ring of integers of $K, L$.

(7) $V_{0} \cong\left(L^{0}\right)^{3}$ : an $L^{0}$-module with on it the unitary form $f(x, y)=x_{1} \bar{y}_{2}+x_{2} \bar{y}_{1}+$ $2 x_{0} \overline{y_{0}}$.

(8) $V:=V_{0} \otimes L:$ on it we have the unitary form $f \otimes L$ which we also denote by $f$. Note that if $\operatorname{Char}(K) \neq 2$, every unitary form on $V$ that gives rise to a unitary group of rank one can be brought into the form $f$ after a suitable choice of the basis. 
(9) $G$ : the linear algebraic group defined over $K^{0}$ that acts on $V_{0}$ preserving $f$. Since we have not defined the module $V_{0}$ over $K^{0}$, the action of $G$ on $V_{0}$ is not defined over $K^{0}$. However one can define a linear action of $G$ on $V_{0} \times V_{0}$ over $K^{0}$ by letting $g \in G$ act as $g \times \tau(g)$ (See also 2.1).

(10) $G\left(K^{0}\right)=S U_{3}\left(L^{0}\right)$ and $G(K)=S U_{3}(L)$.

(11) $S \subset G$ : the torus in $G$ that is diagonal with respect to the coordinates $x_{0}, x_{1}, x_{2}$ of $V_{0}$.

(12) $S(K) \cong K^{*}$ : the maximal $K$-split torus in $G(K)$ coming from $S$.

(13) $Z$ : the centraliser in $G$ of $S$. One has $Z(K) \cong L^{*}$. The subgroup $Z(K) \subset G(K)$ consists of all the elements that act diagonally with respect to the coordinates $x_{0}, x_{1}, x_{2}$.

1.2. The building of $S U_{3}(L)$. The Bruhat-Tits building $B$ of $S U_{3}(L)$ is a tree. We give a combinatorial description of $B$. The vertices of $B$ correspond 1-1 with equivalence classes of certain $L^{0}$-submodules of $V \cong L^{3}$. The equivalence relation is given by:

$$
M \sim N \text { if and only if } \exists\left(\lambda \in L^{*}\right) \quad \text { such that } \quad M=\lambda \cdot N
$$

for $M, N \subset V L^{0}$-modules. One denotes the equivalence class of $M$ by [ $\left.M\right]$.

Let $e_{0}, e_{1}, e_{2}$ be an $L$-basis of $V$ such that the unitary form $f$ has the standard form $f(x, y)=x_{1} \bar{y}_{2}+x_{2} \bar{y}_{1}+2 x_{0} \bar{y}_{0}$ with respect to this basis.

One takes the following two $L^{0}$ submodules in $V$ :

$$
M_{0}:=\left\langle e_{0}, e_{1}, e_{2}\right\rangle, \quad M_{1}:=\left\langle e_{0}, \pi e_{1}, e_{2}\right\rangle .
$$

The building $B$ is given by :

$$
\begin{array}{ll}
\text { vertices: } & S U_{3}(L) \text { images of }\left[M_{0}\right] \text { and }\left[M_{1}\right] \\
\text { edges (or chambers) }: & S U_{3}(L) \text { images of }\left\{\left[M_{0}\right],\left[M_{1}\right]\right\} .
\end{array}
$$

The tree $B$ depends on whether $L / K$ is ramified or not. Let $q:=\sharp \bar{K}$; then $B$ has the following form:

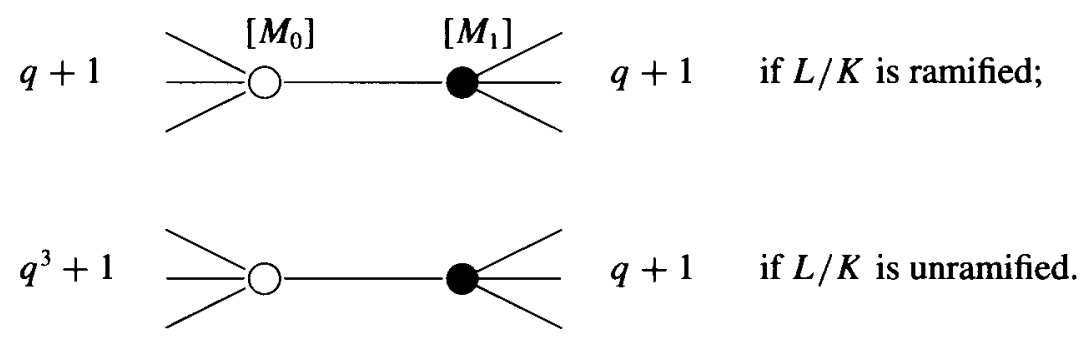


1.3. The root system. The root system of $S U_{3}(L)$ is of type $B C_{1}$. One has for the maximal $K$ split torus $S(K) \cong K^{*}$ four additive groups $U_{ \pm 2 \alpha} \subset U_{ \pm \alpha}$ in $S U_{3}(L)$. The group $U_{\alpha}$ consists of the elements $u_{\alpha}(a, b)$ and $U_{-\alpha}=\left\{u_{-\alpha}(a, b) \in S U_{3}(L)\right\}$ which are as follows:

$$
u_{\alpha}(a, b)\left\{\begin{array} { l } 
{ e _ { 1 } \rightarrow e _ { 1 } } \\
{ e _ { 2 } \rightarrow e _ { 2 } + a e _ { 0 } + b e _ { 1 } } \\
{ e _ { 0 } \rightarrow e _ { 0 } - 2 \overline { a } e _ { 1 } }
\end{array} \quad u _ { - \alpha } ( a , b ) \left\{\begin{array}{l}
e_{1} \rightarrow e_{1}+a e_{0}+b e_{2} \\
e_{2} \rightarrow e_{2} \\
e_{0} \rightarrow e_{0}-2 \bar{a} e_{2} .
\end{array}\right.\right.
$$

In both cases $a$ and $b$ satisfy: $2 a \bar{a}+b+\bar{b}=0$.

Now $U_{2 \alpha} \subset U_{\alpha}$ is $U_{2 \alpha}=\left\{u_{\alpha}(0, b) \mid b+\bar{b}=0\right\}$ and $U_{-2 \alpha}=\left\{u_{-\alpha}(0, b) \mid b+\bar{b}=0\right\}$.

1.4. The affine root system. Let $v$ denote the additive valuation of $L$ with $v(\pi)=1$. One defines the following subgroups of $U_{ \pm \alpha}$ and $U_{ \pm 2 \alpha}$ for $n \in \mathbb{Z}$ :

$$
\begin{aligned}
U_{n+\alpha} & :=\left\{u_{\alpha}(a, b) \in U_{\alpha} \mid v(b) \geq 2 n\right\} ; \\
U_{n+2 \alpha} & :=\left\{u_{\alpha}(0, b) \in U_{2 \alpha} \mid v(b) \geq n\right\} ; \\
U_{n-\alpha} & :=\left\{u_{-\alpha}(a, b) \in U_{-\alpha} \mid v(b) \geq 2 n\right\} ; \\
U_{n-2 \alpha} & :=\left\{u_{-2 \alpha}(0, b) \in U_{-2 \alpha} \mid v(b) \geq n\right\} .
\end{aligned}
$$

Note that $v(b) \geq 2 n$ implies $v(a) \geq n$, since $2 a \bar{a}+b+\bar{b}=0$. If $b+\bar{b}=0$, then $b=c \cdot \gamma$, for $c \in K$ and $\gamma \in L$ fixed such that $\gamma+\bar{\gamma}=0$. If $L / K$ is unramified, one can choose $\gamma$ s.t $v(\gamma)=0$. Hence $\{v(b) \mid b+\bar{b}=0\}=\mathbb{Z}$.

If $L / K$ is ramified one can choose $\gamma$ such that $v(\gamma)=1$. Hence $\{v(b) \mid b+\bar{b}=$ $0\}=\{2 n+1 \mid n \in \mathbb{Z}\}$ in this case.

This gives us the following affine roots for $S(K) \cong K^{*}$ in $S U_{3}(L)$ :

$$
\begin{aligned}
& 2 n+1 \pm 2 \alpha, \quad n \pm \alpha, \quad n \in \mathbb{Z} \quad \text { if } L / K \quad \text { is ramified; } \\
& n \pm 2 \alpha, \quad n \pm \alpha, \quad n \in \mathbb{Z} \quad \text { if } L / K \quad \text { is unramified. }
\end{aligned}
$$

The affine Dynkin diagrams for these root systems are:

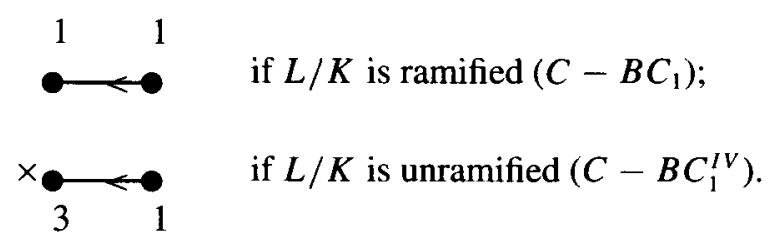

(For more details see $[3, \S 10.1]$ or $[7, \S 1.16]$.) 
1.5. Parahoric subgroups. In the building $B$ there is an apartment $A$ associated to the maximal $K$-split torus $S(K) \cong K^{*}$. Its vertices correspond to $\left[M_{n}\right], n \in \mathbb{Z}$ where $M_{2 n}:=\left\langle e_{0}, \pi^{n} e_{1}, \pi^{-n} e_{2}\right\rangle$ and $M_{2 n+1}:=\left\langle e_{0}, \pi^{n+1} e_{1}, \pi^{-n} e_{2}\right\rangle$. We will denote the vertex in $A$ belonging to $\left[M_{n}\right]$ by $\mathrm{n}$.

One easily sees that $U_{n+2 \alpha}$ stabilizes the vertices $m \leq n$ and $U_{n+\alpha}$ the vertices $m \leq n / 2$. Moreover, $U_{n-2 \alpha}$ stabilizes the vertices $m \geq-n$ and $U_{n-\alpha}$ the vertices $m \geq-n / 2$.

The parahoric subgroups are the stabilizers of the vertices and edges in $B$. They are generated by $Z\left(K^{0}\right)$ and the additive groups stabilizing it.

For the edge $\{0,1\}$ one finds as stabilizer the group generated by $Z\left(K^{0}\right), U_{1+\alpha}$, $U_{0-\alpha}, U_{1+2 \alpha}$ and $U_{0-2 \alpha}$, if $L / K$ is unramified. If $L / K$ is ramified this parahoric subgroup is generated by the groups $Z\left(K^{0}\right), U_{1+\alpha}, U_{0-\alpha}, U_{1+2 \alpha}$ and $U_{1-2 \alpha}$.

1.6. The subgroups $S U_{2}(L) \subset S U_{3}(L)$. The elements $u_{ \pm \alpha}(0, b), b+\bar{b}=0$ generate a subgroup $S U_{2}(L) \subset S U_{3}(L)$. Since $S U_{2}(L) \cong S L_{2}(K)$, this gives an embedding of the $S L_{2}(K)$ building in the building $B$ of $S U_{3}(L)$. Note that both groups have the same rank and that both buildings are trees.

The torus $S(K)$ is contained in $S U_{2}(L)$. It acts on the apartment $A$ belonging to $S(K) \cong K^{*}$. The additive groups for $K^{*}$ in $S U_{2}(L)$ are $U_{ \pm 2 \alpha}$. The associated affine roots are $n \pm 2 \alpha$ if $L / K$ is unramified, and $2 n+1 \pm 2 \alpha$ if $L / K$ is ramified. Hence the vertices of $A$ which are also vertices in the $S L_{2}(K)$ building are $n$ if $L / K$ is unramified and $2 n+1$ if $L / K$ is ramified.

We define $B_{2}:=\bigcup_{g \in S U_{2}(L)} g(A) \subset B$. Then $B_{2} \subset B$ is the $S L_{2}(K)$ building. This embedding $B_{2} \hookrightarrow B$ is simplicial if $L / K$ is unramified. If $L / K$ ramifies, then every $S L_{2}(K)$ chamber consists of two chambers (for $S U_{3}(L)$ in $B$ ).

The embedding $B_{2} \hookrightarrow B$ is unique since a maximal $K$-split torus $K^{*} \subset S U_{2}(L) \subset$ $S U_{3}(L)$ determines a unique apartment $A \subset B$.

The subgroup $S U_{2}(L)$ preserves the decomposition $\left\langle e_{0}>\oplus\left\langle e_{1}, e_{2}>\right.\right.$ of $V$ and the unitary form $x_{1} \overline{y_{2}}+x_{2} \overline{y_{1}}$ on $<e_{1}, e_{2}>$. From this it also follows that the apartment $A \subset B$ is contained in exactly one $S U_{2}(L)$ sub-building.

\section{The action of the torus on $\mathbb{P}_{L}^{2}$}

2.1. Preliminaries. The group $G$ acts on $\mathbb{P}_{L^{0}}^{2} \cong \mathbb{P}\left(V_{0}\right)$. This action is not defined over $K^{0}$, but over $L^{0}$. However, there exists a scheme $\Xi \subset \mathbb{P}\left(V_{0} \times V_{0}\right)$ defined over $K^{0}$ such that $\Xi \otimes L^{0}=\mathbb{P}\left(V_{0} \times<0>\right) \cup \mathbb{P}\left(<0>\times V^{0}\right) \cong \mathbb{P}_{L^{0}}^{2} \cup \mathbb{P}_{L^{0}}^{2}$. The action of $G$ on $\Xi$ is defined over $K^{0}$. The group $\operatorname{Gal}(L / K)$ permutes the two components $\mathbb{P}_{L^{0}}^{2}$.

The torus $S \subset G$ acts on $X:=\mathbb{P}\left(V_{0}\right) \cong \mathbb{P}_{L^{0}}^{2}$. This action is defined over $L^{0}$. We 
make the following definitions:

$$
\begin{aligned}
X_{S}^{s s} & :=\text { set of semistable points for } S=\left\{x \in \mathbb{P}_{L^{0}}^{2} \mid x_{0}^{2} \text { or } x_{1} x_{2} \text { is invertible }\right\}, \\
X_{S}^{s} & :=\text { set of stable points for } S=\left\{x \in \mathbb{P}_{L^{0}}^{2} \mid x_{1} x_{2} \text { is invertible }\right\} .
\end{aligned}
$$

Note that the centraliser $Z\left(K^{0}\right) \cong\left(L^{0}\right)^{*}$ of $S\left(K^{0}\right)$ acts on both spaces.

Let $f$ be a homogeneous polynomial of degree $n$. Then $f$ is called invertible at $x \in X_{S}^{s s}$ if $|f(x)|=\max \left\{\left|x_{i}\right|^{n} \mid i=0,1,2\right\}$. In particular this means that $f \neq 0$ on the closed fibre of $X_{S}^{s s}$. Furthermore one has:

$$
\begin{aligned}
X_{S}^{s s} \otimes L & =\left\{x \in \mathbb{P}_{L}^{2} \mid x_{0}^{2} \neq 0 \quad \vee \quad x_{1} x_{2} \neq 0\right\}=\mathbb{P}_{L}^{2}-\{(0,1,0),(0,0,1)\}, \\
X_{S}^{s} \otimes L & =\left\{x \in \mathbb{P}_{L}^{2} \mid x_{1} x_{2} \neq 0\right\}=\mathbb{P}_{L}^{2}-\left\{\left(x_{0}, x_{1}, 0\right),\left(x_{0}, 0, x_{2}\right)\right\} .
\end{aligned}
$$

2.2. Analytifications. To each algebraic variety corresponds a rigid analytic variety which has the same set of closed points (See [2] or [1]). We denote the analytic varieties corresponding to $X_{S}^{s} \otimes L$ and $X_{S}^{s s} \otimes L$ by $Y_{A}^{s}$ and $Y_{A}^{s s}$ respectively. Here $A$ is the apartment in $B$ belonging to $S(K)$.

We also need some analytic spaces corresponding to $X_{S}^{s s}$ and $X_{S}^{s}$. The set of points of these spaces consists of their closed fibres. They are:

$$
\begin{aligned}
Y_{0, A}^{s s}:= & \text { the completion of } X_{S}^{s s} \text { along the closed fibre } \\
= & \left\{x \in Y_{A}^{s s}|| x_{1} x_{2} / x_{0}^{2}|\leq 1,| x_{1} / x_{0}|\leq 1,| x_{2} / x_{0} \mid \leq 1\right\} \\
& \cup\left\{x \in Y_{A}^{s s}|| x_{0}^{2} / x_{1} x_{2}|\leq 1,| x_{1} / x_{2} \mid=1\right\}, \\
Y_{0, A}^{s}:= & \text { the completion of } X_{S}^{s} \text { along the closed fibre } \\
= & \left\{x \in Y_{A}^{s s}|| x_{0}^{2} / x_{1} x_{2}|\leq 1,| x_{1} / x_{2} \mid=1\right\} .
\end{aligned}
$$

Here the suffix 0 corresponds to the vertex $0 \in A$. In fact we need similar analytic subspaces for every simplex, that is, vertex or edge, $\sigma \in A$. This is done as in [4, $\S 3.3, \S 3.4]$.

First we analytify the torus $S \otimes K$. From now on $S$ will denote the analytification of $S \otimes K$. For each simplex $\sigma \in A$ one defines the affinoid subspace $S_{\sigma} \subset S$ by:

$$
\left.S_{\sigma}:=\operatorname{Sp}\left(K\left\langle\pi^{n} \chi\right| n \in \mathbb{Z}, \quad \chi \in \chi(S), \quad n+\chi \geq 0 \quad \text { on } \sigma\right\rangle\right) .
$$

For this definition one has to identify the apartment $A$ with the dual of $\chi(S) \otimes \mathbb{R}$. Equivalently one can also define $S_{\sigma}$ by $S_{\sigma}:=\{s \in S \mid s \cdot 0 \in \sigma\}$. Here one identifies $A \cong \mathbb{R}$, and $s \in S$ acts on $A$ by translation by $2 \cdot v\left(s_{1}\right)$.

Our torus $S$ has, with respect to the coordinates $x_{0}, x_{1}, x_{2}$, a diagonal form $s=$ $\operatorname{diag}\left(s_{0}, s_{1}, s_{2}\right)$ with $s_{0}=1, s_{1} s_{2}=1$. Hence one may put $s_{1}=t, s_{2}=t^{-1}$ and $s_{0}=1$. For the standard chamber $\sigma_{0}=\{0,1\}=\left\{\left[M_{0}\right],\left[M_{1}\right]\right\}$ one has

$$
S_{\sigma_{0}}=\operatorname{Sp}\left(K\left\langle t, \pi t^{-2}\right\rangle\right) \text {. }
$$


As in [4] one defines:

$$
Y_{\sigma, A}^{s}:=S_{\sigma} \cdot Y_{0, A}^{s}=\left\{s \cdot x \mid s \in S_{\sigma}, x \in Y_{0, A}^{s}\right\} ; \quad Y_{\sigma, A}^{s s}:=S_{\sigma} \cdot Y_{0, A}^{s s} .
$$

Note that in [4] one restricts to the case where the variety $X$ satisfies $X^{s}=X^{s s}$. Therefore the spaces $Y_{\sigma, A}^{s}$ and $Y_{\sigma, A}^{s s}$ coincide. Since in our case $Y_{\sigma, A}^{s} \neq Y_{\sigma, A}^{s s}$, we cannot use these spaces to construct an affinoid covering of $Y_{A}^{s}$. For that we need to do a little more.

First one needs a definition.

DEFINITION 2.3. For $x \in Y_{A}^{s s}$ we define the interval of $S$ semistability by:

$$
I_{A}(x):=\overline{\left\{s^{-1} \cdot 0 \mid s \cdot x \in Y_{0, A}^{s s}, s \in S\right\}} \subset A
$$

Here $s \in S$ means $S \in S\left(K^{\mathrm{alg}}\right)$, where $K^{\text {alg }}$ is the algebraic closure of $K$. Hence if one puts $A \cong \mathbb{R}$, the points $s^{-1} \cdot 0$ are in $\mathbb{Q}$. This is the reason one takes the closure $\overline{\left\{s^{-1} \cdot 0 \mid \ldots\right\}}$ instead of just $\{\ldots\}$.

This map $I_{A}$ which associates to a point $x \in Y_{A}^{s s}$ a subset of $A$ replaces the function $v_{X . T . L}: Y_{A}^{s s} \rightarrow A$ used in [4]. One has:

PRoposition 2.4. Let $x \in Y_{A}^{s s}$. Then:

(1) $I_{A}(s \cdot x)=s \cdot I_{A}(x)$ for all $s \in S$.

(2) $I_{A}(x) \subset A$ is convex.

(3) $I_{A}(x)=A$ if and only if $x=(1,0,0)$.

(4) $I_{A}(x) \subset A$ is a half-apartment if and only if $x \in Y_{A}^{s s}-Y_{A}^{s}, x \neq(1,0,0)$.

(5) $I_{A}(x) \subset A$ is bounded if and only if $x \in Y_{A}^{s}$.

PROOF. Part 1 follows directly from the definition of $I_{A}(x)$. To prove the other statements, we will describe $I_{A}(x)$ for all $x \in Y_{A}^{s s}$.

If $x=(1,0,0)$ then $x \in Y_{0, A}^{s s}$. Furthermore $x$ is a fixed point for $S$. This proves $I_{A}(x)=A$.

If $x \in Y_{A}^{s s}$ and $\left|x_{0}^{2}\right| \leq\left|x_{1} x_{2}\right|$, then $x \in Y_{A}^{s}$. We may assume $\left|x_{1}\right|=\left|x_{2}\right|$ after replacing $x$ by $s \cdot x$ for suitable $s \in S$. Now $x \in Y_{0, A}^{s}$ and one easily sees that $I_{A}(x)$ consist only of the point 0 .

If $x=\left(x_{0}, x_{1}, 0\right)$ with $x_{0}, x_{1} \neq 0$, then by using 1 we may assume $\left|x_{0}\right|=\left|x_{1}\right|$. Then $s \cdot x \in Y_{0, A}^{s s}$ if and only if $\left|s_{1}\right| \leq 1$. So $I_{A}(x)$ is a half-apartment in this case. The case $x=\left(x_{0}, 0, x_{2}\right)$ is similar.

The only points in $Y_{A}^{s s}$ not yet treated are those with $\left|x_{1} x_{2}\right|<\left|x_{0}^{2}\right|$ and $x_{1} x_{2} \neq 0$. It is enough to treat those $x$ with $\left|x_{1}\right|=\left|x_{2}\right|<\left|x_{0}\right|$. Now $s \cdot x \in Y_{0, A}^{s s}$ if and only if $\left|s_{1} x_{1}\right| \leq\left|x_{0}\right|$ and $\left|s_{2} x_{2}\right| \leq\left|x_{0}\right|$. Since $\left|s_{1}\right|=\left|s_{2}^{-1}\right|$ one finds $\left|x_{2} / x_{0}\right| \leq\left|s_{1}\right| \leq\left|x_{0} / x_{1}\right|$ in this case. Hence $I_{A}(x)$ is a bounded interval in $A$. 
We now have treated all points $x \in Y_{A}^{s s}$. One easily verifies that statements 2 to 5 hold.

Proposition 2.5. For $x \in Y_{A}^{s s}$ one has:

(1) $x \in Y_{\sigma, A}^{s s}$ if and only if $I_{A}(x) \cap \sigma \neq \emptyset$.

(2) $x \in Y_{\sigma, A}^{s}$ if and only if $I_{A}(x)$ is a point contained in $\sigma$.

PROOF. Part 1 follows directly from the definitions. Part 2 follows from $x \in Y_{0, A}^{s}$ if and only if $I_{A}(x)=\{0\}$.

REMARK 2.6. The space $Y_{\sigma, A}^{s}$ is affinoid, but $Y_{\sigma, A}^{s s}$ is not. One can cover $Y_{\sigma, A}^{s s}$ by the affinoid subspaces $Y_{\sigma, A}^{s}$ and $Y_{\sigma, A}^{s s+}$. Here $Y_{\sigma, A}^{s s+}:=\left\{x \in Y_{\sigma, A}^{s s}|| x_{1} x_{2} / x_{0}^{2} \mid \leq 1\right\}$. The covering $\left\{Y_{\sigma, A}^{s}, Y_{\sigma, A}^{s s+}\right\}$ of $Y_{\sigma, A}^{s s}$ is pure.

Let $\mathscr{C}_{A}^{s}:=\left\{Y_{\sigma, A}^{s} \mid \sigma \in A\right\}$ and $\mathscr{C}_{A}^{s s}:=\left\{Y_{\sigma, A}^{s}, Y_{\sigma, A}^{s s+} \mid \sigma \in A\right\}$. In the figure below we draw the covering $\mathscr{C}_{A}^{s s}$ using the values of $v\left(x_{i} / x_{j}\right)$, where $v$ denotes the valuation of $L$.

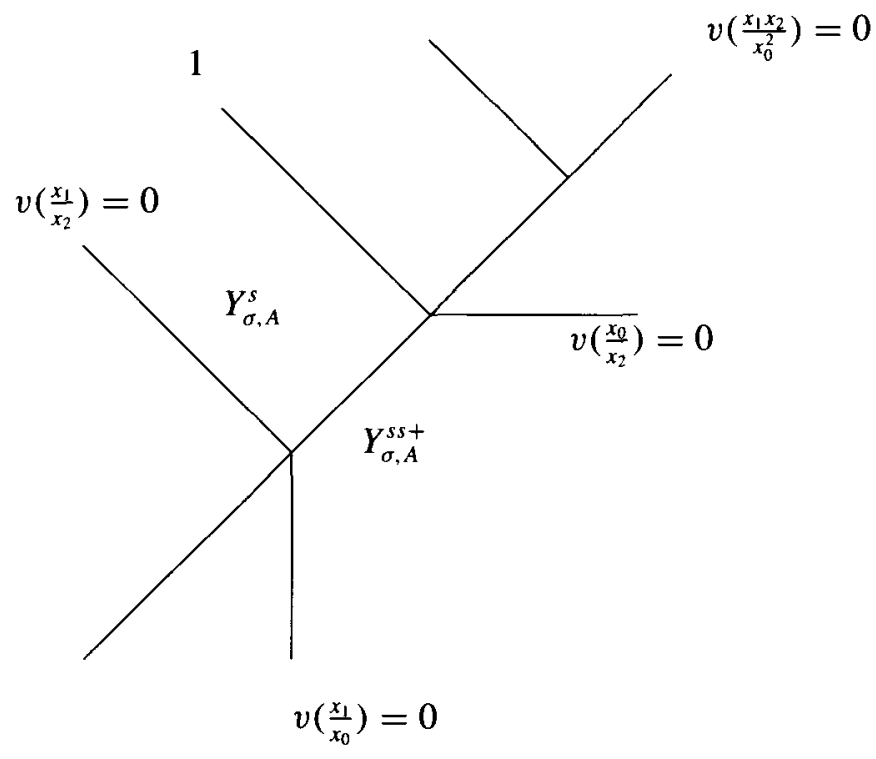

PROPOSITION 2.7.

(1) (a) $\bigcup_{\sigma \in A} Y_{\sigma, A}^{s}=\left\{x \in Y_{A}^{s s} \mid I_{A}(x)\right.$ is a point $\}=\left\{x \in Y_{A}^{s s}|| x_{0}^{2} / x_{1} x_{2} \mid \leq 1\right\} \underset{\neq}{\complement} Y_{A}^{s}$.

(b) The covering $\mathscr{C}_{A}^{s}$ is pure. 
(c) The components of the reduction of $\bigcup_{\sigma \in A} Y_{\sigma, A}^{s}$ with respect to the covering $\mathscr{C}^{s}$ are not proper.

(2) (a) $\bigcup_{\sigma \in A} Y_{\sigma, A}^{s s}=Y_{A}^{s s}$.

(b) The covering $\mathscr{C}_{A}^{s s}$ of $Y_{A}^{s s}$ is not pure.

PROOF. Parts 1(a) and 2(a) are a direct consequence from the previous proposition. The other parts of the proposition follow more or less immediately from the picture of the covering $\mathscr{C}_{A}^{s s}$ given above, using $[8, \S 2]$. Note that $S$ translates along the line $v\left(x_{0}^{2} / x_{1} x_{2}\right)=0$ in the picture above.

REMARK 2.8. The proposition above shows that one cannot use the affinoids $Y_{\sigma, A}^{s}$ and $Y_{\sigma, A}^{s s+}$ to get a good affinoid covering of $Y_{A}^{s}$ or $Y_{A}^{s s}$. Before we give a pure affinoid covering of $Y_{A}^{s}$ we need to know $I_{A}(x)$ in more detail. First we need a definition.

DEFINITION 2.9. For $x \in Y_{A}^{s}$ the interval $I_{A}(x)$ has two extremal points $P_{1}$ and $P_{2}$. There exists a unique point $P_{3} \in A$ which has equal distance to both $P_{1}$ and $P_{2}$. Hence one can define:

$$
v_{A}(x):=P_{3}=\left(P_{1}+P_{2}\right) / 2 .
$$

Note that one cannot extend $v_{A}$ to $Y_{A}^{s s}$.

As before $v$ will denote the additive valuation of $L$, such that $v(\pi)=1$, extended to the algebraic closure of $L$ (or $K$ ). Also we identify $A$ with $\mathbb{R}$ such that the vertices correspond to the integers as before. The interval with extremal points $P_{1}$ and $P_{2}$ will be denoted by $\left[P_{1}, P_{2}\right]_{A}:=$ convex hull of $\left\{P_{1}, P_{2}\right\}$. Using this definition we obtain:

PROPOSITION 2.10. For $x \in Y_{A}^{s}$ one has: $v_{A}(x)=v\left(x_{1} / x_{2}\right)$ and

$$
I_{A}(x)=\left\{\begin{array}{lll}
{\left[2 v\left(x_{0} / x_{2}\right), 2 v\left(x_{1} / x_{0}\right)\right]_{A}} & \text { if } & \left|x_{1} x_{2} / x_{0}^{2}\right| \leq 1, \\
\left\{v_{A}(x)\right\} & \text { if }\left|x_{0}^{2} / x_{1} x_{2}\right| \leq 1 .
\end{array}\right.
$$

ProOF. First we remark that $s \in S$ acts on $A$ by translation with $v\left(s_{1} / s_{2}\right)=2 v\left(s_{1}\right)$. So the descriptions in the proposition satisfy $v_{A}(s \cdot x)=s \cdot v_{A}(x)$ and $I_{A}(s \cdot x)=s \cdot I_{A}(x)$ as they should.

Hence it is sufficient to prove the theorem for $x \in Y_{A}^{s}$ with $\left|x_{1}\right|=\left|x_{2}\right|$. If $\left|x_{1} x_{2}\right| \geq\left|x_{0}^{2}\right|$ then $I_{A}(x)=\{0\}$. Hence $v_{A}(x)=0$ and the proposition holds in this case.

If $\left|x_{1} x_{2}\right| \leq\left|x_{0}^{2}\right|$ and $\left|x_{1}\right|=\left|x_{2}\right|$, then $s \in S$ with $s \cdot x \in Y_{0 . A}^{s s}$ satisfy $\left|s_{1} x_{1}\right| \leq$ $\left|x_{0}\right|,\left|s_{2} x_{2}\right| \leq\left|x_{0}\right|$. Hence one has $\left|x_{2} / x_{0}\right| \leq\left|s_{1}\right| \leq\left|x_{0} / x_{1}\right|$. Now $s^{-1} \cdot 0 \in$ $I_{A}(x)$. Hence one finds $v\left(x_{1} / x_{0}\right) \geq v\left(s_{1}^{-1}\right) \geq v\left(x_{0} / x_{2}\right)$. Hence one has $I_{A}(x)=$ $\left[2 v\left(x_{0} / x_{2}\right), 2 v\left(x_{1} / x_{0}\right)\right]_{A}$ in this case. 
COROLlaRY 2.11. For $x \in Y_{A}^{s}$ one has:

$$
I_{A}(x)=\left\{z \in A \mid \operatorname{dist}\left(v_{A}(x), z\right) \leq \max \left(0, v\left(x_{1} x_{2} / x_{0}^{2}\right)\right)\right\} .
$$

PROOF. If $v\left(x_{1} x_{2} / x_{0}^{2}\right) \leq 0$ then $I_{A}(x)$ is the point $v_{A}(x)$. Hence the proposition holds in this case.

If $v\left(x_{1} x_{2} / x_{0}^{2}\right) \geq 0$, then $I_{A}(x)=\left[2 v\left(x_{0} / x_{2}\right), 2 v\left(x_{1} / x_{0}\right)\right]_{A}$ and $v_{A}(x)=v\left(x_{1} / x_{2}\right)$. Now $2 v\left(x_{1} / x_{0}\right)-v\left(x_{1} / x_{2}\right)=v\left(x_{1}^{2} / x_{0}^{2} \cdot x_{2} / x_{1}\right)=v\left(x_{1} x_{2} / x_{0}^{2}\right)$. So the proposition also holds in this case.

\section{A pure affinoid covering of $Y_{A}^{s}$}

3.1. Since the 'natural' affinoids $Y_{\sigma, A}^{s}$ do not cover all of $Y_{A}^{s}$ we have to construct some additional affinoids. One wants the covering to be $Z(K)$ invariant.

Such affinoids can be constructed in the following way. Let $\phi:\left\{x \in Y_{A}^{s} \mid\right.$ $\left.x_{0} \neq 0\right\} \rightarrow \mathbb{R}^{2}$ be the map $x \rightarrow\left(v\left(x_{0} / x_{1}\right), v\left(x_{0} / x_{2}\right)\right)$. The inverse image of a bounded convex polyhedron in $\mathbb{R}^{2}$ whose faces are contained in rational lines is an affinoid subspace of $Y_{A}^{s}$. In particular if one covers $\mathbb{R}^{2}$ by polyhedra as above such that the intersection of two such polyhedra is a face of both and such that each 0 dimensional face (that is, vertex) is contained in only finitely many polyhedra, then the corresponding affinoid covering will be pure (See [8, Lemma 2.4]).

In the figure below we give the polyhedra in $\mathbb{R}^{2}$ corresponding to the affinoid covering $\mathscr{C}_{A}$ of $Y_{A}^{s}$ we will use.

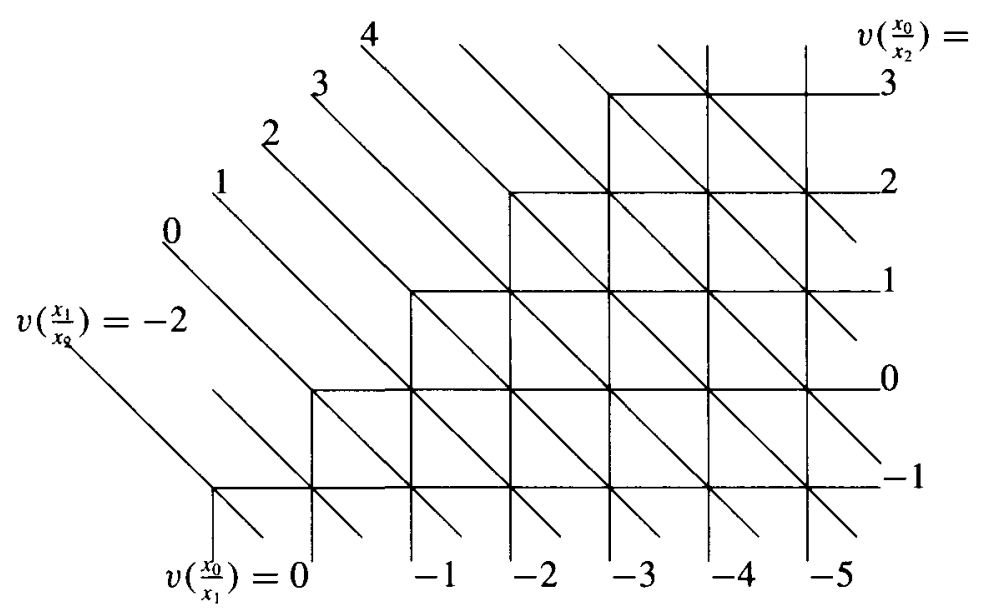

If one knows the point $\phi(x)$ for $x \in Y_{A}^{s}$, then one can easily determine $v_{A}(x)$ and $I_{A}(x)$. In fact, for $x, y \in Y_{A}^{s}$ with $\left|x_{1} x_{2} / x_{0}^{2}\right| \leq 1$ one has $\phi(x)=\phi(y)$ if and only if $I_{A}(x)=I_{A}(y)$. 
The affinoid covering $\mathscr{C}_{A}$ consists of the following affinoids:

$$
\begin{gathered}
Y\left\{\rho_{1}, \rho_{2}, \sigma\right\}_{A}:=\left\{x \in Y_{A}^{s} \mid I_{A}(x) \subset \text { convex hull of }\left\{\rho_{1}, \rho_{2}\right\},\right. \\
\left.I_{A}(x) \cap \rho_{i} \neq \emptyset, i=1,2, v_{A}(x) \in \sigma\right\} .
\end{gathered}
$$

Here $\sigma \in A$ is a chamber and $\rho_{i} \subset A$ is the union of two neighbouring chambers bounded by two vertices of type 0 , that is, $\rho_{i}:=\left[2 n_{i}, 2 n_{i}+2\right]_{A}, n_{i} \in \mathbb{Z}$. For notational reasons we will write $\rho_{i} \in A$ for $\rho_{i} \subset A$. We only consider triples $\rho_{1}, \rho_{2}, \sigma$ such that $Y\left\{\rho_{1}, \rho_{2}, \sigma\right\}_{A}$ is non-empty. Once one fixes $\rho_{1}$ and $\rho_{2}$ there are exactly four choices for $\sigma$ such that $Y\left\{\rho_{1}, \rho_{2}, \sigma\right\}_{A}$ is non-empty. Of these four choices, two correspond to polyhedra in the picture above. The other two correspond to one-dimensional faces if $\rho_{1}=\rho_{2}$ and to vertices if $\rho_{1} \neq \rho_{2}$. By allowing $\sigma$ to be a vertex one can obtain the faces of the polyhedra in the skew lines. If one allows one of the $\rho_{i}$ to be a vertex of type 0 , one obtains the faces of the polyhedra that are contained in the horizontal and vertical lines in the picture above.

Note that if $\sigma \subset \rho$ one has:

$$
Y\{\rho, \rho, \sigma\}_{A}=\left\{x \in Y_{A}^{s} \mid I_{A}(x) \subset \rho, v_{A}(x) \in \sigma\right\} \supset Y_{\sigma, A}^{s} .
$$

The reason that one uses the subsets $\rho_{i} \subset A$, instead of chambers in the definition of the affinoids, is that this choice associates $L^{0}$-submodules of $L^{3}$ to the vertices of the polyhedra in the picture. Had we used chambers, those modules would only have been defined over a finite extension of $L^{0}$. Our choice will therefore ensure that the components of the reduction of $Y^{s}$ will correspond to $L^{0}$-modules (See also Remark 8.11).

In the proposition below we state all the relevant properties of our affinoid covering $\mathscr{C}_{A}$.

PROPOSITION 3.2. (1) $\bigcup_{\rho_{1}, \rho_{2} \in A} Y\left\{\rho_{1}, \rho_{2}, \sigma\right\}=\left\{x \in Y_{A}^{s} \mid v_{A}(x) \in \sigma\right\}$.

(2) $\bigcup_{\rho_{1}, \rho_{2}, \sigma \in A} Y\left\{\rho_{1}, \rho_{2}, \sigma\right\}=Y_{A}^{s}$.

(3) The affinoid covering $\mathscr{C}_{A}=\left\{Y\left\{\rho_{1}, \rho_{2}, \sigma\right\}_{A} \mid \rho_{1}, \rho_{2}, \sigma \in A\right\}$ is pure.

(4) The components of the reduction of $Y_{A}^{s}$ with respect to $\mathscr{C}_{A}$ are proper.

(5) There is a 1-1 correspondence between components of the reduction and integer intervals $[2 n, 2 m]_{A} \subset A, n, m \in \mathbb{Z}$.

PROOF. Statements (1) and (2) are obvious. The statements (3) and (4) are proved as in $[8, \S 2]$. The last statement of the proposition follows from the fact that the extremal points of the polyhedra in the figure above correspond with the integer intervals $[2 n, 2 m]_{A}$ in $A$. 


\section{The action of $S U_{3}(L)$ on $\mathbb{P}_{L}^{2}$}

4.1. In this section we study the affinoids $Y_{\sigma}^{s}$ and $Y_{\sigma}^{s s}$. We then define an interval $I(x)$ of semistability for $x$ with respect to $S U_{3}(L)$, and study the connection between $Y_{\sigma}^{s s}$ and $I(x)$ for $x \in Y^{s s}$. Analogous to $[4, \S 3.5,3.6]$, one defines:

DEFINITION 4.2. $Y^{s}:=\bigcap_{g \in S U_{3}(L)} g\left(Y_{A}^{s}\right)$ and $Y^{s s}:=\bigcap_{g \in S U_{3}(L)} g\left(Y_{A}^{s s}\right)$ Let $A^{\prime} \subset B$ be an apartment and $\sigma^{\prime} \in A^{\prime}$ a simplex. One can find $\sigma \in A$ and $g \in S U_{3}(L)$ such that $g(\sigma)=\sigma^{\prime}$ and $g(A)=A^{\prime}$. Then one takes:

$$
Y_{\sigma^{\prime}, A^{\prime}}^{s}:=g\left(Y_{\sigma, A}^{s}\right) \quad \text { and } \quad Y_{\sigma^{\prime}, A^{\prime}}^{s s}:=g\left(Y_{\sigma, A}^{s s}\right) .
$$

Moreover one needs:

$$
Y_{\sigma}^{s}:=\bigcap_{A^{\prime} \ni \sigma} Y_{\sigma, A^{\prime}}^{s} \quad \text { and } \quad Y_{\sigma}^{s s}:=\bigcap_{A^{\prime} \ni \sigma} Y_{\sigma, A^{\prime}}^{s s}
$$

As in [4] the subspaces $Y_{\sigma}^{s} \subset Y_{\sigma, A}^{s}$ and $Y_{\sigma}^{s s} \subset Y_{\sigma, A}^{s s}$ are nice open subdomains.

DEFINITION 4.3. As in $[4, \S 3.6]$ we define a function $r_{A_{1}, A_{2}}$, which is useful for studying the affinoids defined above. Let $A_{1}=g_{1}(A)$ and $A_{2}=g_{2}(A)$ with $g_{i} \in$ $S U_{3}(L)$. For $z \in \mathbb{P}_{L}^{2}$ define:

$$
r_{A_{1}, A_{2}}(z):=\max \left\{\left|g_{1}^{*} x_{1} g_{1}^{*} x_{2}(z)\right|,\left|g_{1}^{*} x_{0}^{2}(z)\right|\right\} / \max \left\{\left|g_{2}^{*} x_{1} g_{2}^{*} x_{2}(z)\right|,\left|g_{2}^{*} x_{0}^{2}(z)\right|\right\} .
$$

This function now has, mutatis mutandis, the same properties in our situation as in [4].

In the lemma below we state the properties of $r_{A_{1}, A_{2}}$ which are either obvious or for which the proof is exactly as in $[4, \S 3.6]$.

LEMMA 4.4. (a) $r_{g A, A}(x)$ is well defined for $x \in Y_{A}^{s s}$.

(b) $r_{g A, A}(x)$ only depends on $A$ and $g A$ and not on the choice of $g \in S U_{3}(L)$.

(c) $r_{g h A, h A}(x) \cdot r_{h A, A}(x)=r_{g h A, A}(x)$ and $r_{g A, A}(x)=\left(r_{A, g A}(x)\right)^{-1}$.

(d) $x \in Y_{\sigma, A}^{s s}$ implies $r_{g A, A}(x) \leq 1 \forall\left(g \in P_{\sigma}\right)$.

Here $P_{\sigma}$ denotes the stabiliser of $\sigma$ in $S_{3}(L)$.

DEFINITION 4.5. Define:

$$
r(x):= \begin{cases}0 & \text { if } x \notin Y_{A}^{s s}, \\ \inf \left\{r_{g A, A}(x) \mid g \in G(K)\right\} & \text { if } x \in Y_{A}^{s s} .\end{cases}
$$

One has:

PROPOSITION 4.6. (a) $x \in Y_{\sigma, g A}^{s s}$ and $r_{g A, A}(x)=r(x)>0$ if and only if $x \in Y_{\sigma}^{s s}$. 
(b) $x \in Y^{s s}$ if and only if $r(x)>0$.

Proof. The proofs of similar statements in $[4,3.6(\mathrm{~d})$, (f)] remain valid in our case, mutatis mutandis.

DEFINITION 4.7. To understand the analytic space $Y^{s s}$ better, define, for $x \in Y^{s s}$, the interval of $\mathrm{SU}_{3}(L)$-semistability by

$$
I(x):=\left\{z \in B \mid \forall(A \ni z) z \in I_{A}(x)\right\} \subset B .
$$

Here $I_{g A}(x), g \in S U_{3}(L)$, is defined by $I_{g A}(x):=g\left(I_{A}\left(g^{-1}(x)\right)\right)$. This is well defined, since $t\left(I_{A}\left(t^{-1}(x)\right)\right)=I_{A}(x)$ for $t \in Z(K)$.

From the definition one gets:

$$
0 \in I(x) \text { if and only if } x \in Y_{0}^{s s} .
$$

A close look at the proof of in $[4,3.6(\mathrm{f})]$ gives us:

PROPOSITION 4.8. $x \in Y_{\sigma}^{s s}$ if and only if

$$
\forall\left(A_{1}, A_{2} \ni \sigma\right) \quad I_{A_{1}}(x) \cap \sigma=I_{A_{2}}(x) \cap \sigma \neq \emptyset .
$$

PROOF. It is sufficient to proof it for $\sigma \in A$ a chamber. Let $z \in I_{A}(x) \cap \sigma$; since $x \in Y_{\sigma, A}^{s s}$, such a $z$ exist. It is now sufficient to prove, for all $z \in I_{A}(x) \cap \sigma$, that $z \in I_{g A}(x) \cap \sigma$ for all $g \in P_{\sigma}$. It is sufficient to prove it only in case $z \in \mathbb{Q} \subset A \cong \mathbb{R}$.

We take a finite extension $K^{\prime} \supset K$ such that $z \in 2 v\left(\left(K^{\prime}\right)^{*}\right)$. Now there exists an element $s \in S\left(K^{\prime}\right) \cong\left(K^{\prime}\right)^{*}$ such that $z=s^{-1} \cdot 0$. We put $Y_{z, A}^{s s}:=s^{-1} \cdot\left(Y_{0, A}^{s s} \otimes K^{\prime}\right)$.

Clearly we have $z \in I_{A}(x)$ if and only if $x \in Y_{z, A}^{s s}$.

We put $Y_{z}^{s s}:=\bigcap_{g \in P_{\sigma}} g\left(Y_{z, A}^{s s}\right)$. Since $r_{g A, A}(x) \leq 1, \forall g \in P_{\sigma}$, and $r_{A, A}(x)=r(x)=$ 1 by Proposition 4.6(a), we must have $r_{g A, A}(x)=1$ for all $g \in P_{\sigma}$.

Hence $x \in Y_{z}^{s s}$. In particular $x \in Y_{z, g A}^{s s}$ and therefore $z \in I_{g A}(x)$ for all $g \in P_{\sigma}$. This proves the proposition.

PROPOSITION 4.9. (1) $x \in Y_{\sigma}^{s s}$ if and only if $I(x) \cap \sigma \neq \emptyset$;

(2) Let $R(x):=\left\{A^{\prime} \subset B \mid r_{A^{\prime}, A}(x)=r(x)\right\}$. For $x \in Y^{s s}$ one has:

$$
I(x)=\bigcup_{A^{\prime} \subset R(x)} I_{A^{\prime}}(x) .
$$

(3) $x \in Y_{\sigma}^{s}$ if and only if $I(x)$ is a point contained in $\sigma$.

PROOF. The first statement follows directly from Proposition 4.8. The second statement is a direct consequence of Propositions 4.8 and 4.6. The third part is clear from Definition 4.7. 
COROLlaRY 4.10. (1) $I(x)$ is convex.

(2) $x \in Y^{s}$ if and only if $I(x)$ is bounded.

We will omit the proof of this corollary, since it is also a trivial consequence of Theorem 6.2 below. Now we can state what remains true of [4, Theorem 3.6] in our case.

PROPOSITION 4.11. (1) $Y^{s s}=\bigcup_{\sigma \in B} Y_{\sigma}^{s s}$.

(2) (a) $Y_{\sigma_{1}}^{s} \cap Y_{\sigma_{2}}^{s}=\emptyset$ if $\sigma_{1} \cap \sigma_{2}=\emptyset$ and equals $Y_{\sigma_{3}}^{s}$ if $\sigma_{1} \cap \sigma_{3}=\sigma_{3}$.

(b) $Y_{\sigma_{1}}^{s s} \cap Y_{\sigma_{2}}^{s s} \neq \emptyset \quad \forall \sigma_{1}, \sigma_{2} \in B$.

(3) The covering $\mathscr{C}^{s}:=\left\{Y_{\sigma}^{s} \mid \sigma \in B\right\}$ is pure. It covers the space

$$
\left\{x \in Y^{s} \mid I(x) \text { is a point }\right\} \underset{\neq}{\subsetneq} Y^{s} .
$$

PROOF. Except for 2(b), everything follows easily from the previous propositions. As for 2(b), we note that for $\sigma_{1}, \sigma_{2} \in B$ one can find $x$ such that $I(x) \cap \sigma_{1} \neq \emptyset$ and $I(x) \cap \sigma_{2} \neq \emptyset$ as follows.

Clearly if $\sigma_{1}, \sigma_{2} \in A$ one can find $x$ such that $\sigma_{1} \cap I_{A}(x) \neq \emptyset$ and $\sigma_{2} \cap I_{A}(x) \neq \emptyset$. By choosing $x$ carefully one may assume that $A \subset R(x)$. Hence $I_{A}(x) \subset I(x)$. So we have constructed a point $x \in Y_{\sigma_{1}}^{s s} \cap Y_{\sigma_{2}}^{s s}$.

\section{The action of $S U_{2}(L)$ on $\mathbb{P}_{L}^{2}$}

5.1. Before determining the interval of $S U_{3}(L)$ semistability for $x \in Y^{s s}$ it is useful to study the interval of semistability with respect to the subgroup $S U_{2}(L) \subset$ $S U_{3}(L)$. One has an $S U_{2}\left(L^{0}\right)$-equivariant map $\varphi: \mathbb{P}_{L^{0}}^{2}-\{(1,0,0)\} \rightarrow \mathbb{P}_{L^{0}}^{1}$, given by $\varphi\left(x_{0}, x_{1}, x_{2}\right)=\left(x_{1}, x_{2}\right)$. One can use the action of $S U_{2}(L)$ on $\mathbb{P}_{L}^{1}$ to study the action of $S U_{2}(L)$ on $\mathbb{P}_{L}^{2}$. Since $S U_{2}(L) \cong S L_{2}(K)$ the space of points in $\mathbb{P}_{L}^{1}$ which are stable for all maximal $K$-split tori in $S U_{2}(L)$ is essentially Mumford's upper halfplane $\Omega_{1}$.

5.2. The action of $S U_{2}(L)$ on $\mathbb{P}_{L^{1}}^{1}$. Let $S U_{2}\left(L^{0}\right)$ act on $\mathbb{P}_{L^{0}}^{1}$ respecting the unitary form $\tilde{f}(x, y)=x_{1} \bar{y}_{2}+x_{2} \bar{y}_{1}$. For the torus $T \subset S U_{2}\left(L^{0}\right)$ acting diagonally for the coordinates $x_{1}, x_{2}$, the sets of stable and semistable points $\left(\mathbb{P}^{1}\right)_{T}^{s}$ and $\left(\mathbb{P}^{1}\right)_{T}^{s s}$ coincide, that is, $\left(\mathbb{P}^{1}\right)_{T}^{s}=\left(\mathbb{P}^{1}\right)_{T}^{s s}$. In particular, all the results of [4] apply to our situation.

Let $B_{2}$ be the building of $S U_{2}(L) \cong S L_{2}(K)$ and $A \subset B_{2}$ the apartment belonging to $T(K)$. Let $\mathscr{Z}_{A}$ be the analytic space corresponding to $\left(\mathbb{P}_{L^{0}}^{1}\right)_{T}^{s s} \otimes L$ and $\mathscr{Z}_{0, A}$ the completion of $\left(\mathbb{P}^{1}\right)_{T}^{s s}$ along the closed fibre.

One has: $\quad \mathscr{Z}_{A}=\left\{x \in \mathbb{P}_{L}^{1} \mid x_{1} \neq 0 \wedge x_{2} \neq 0\right\}$,

$$
\mathscr{Z}_{0, A}=\left\{x \in \mathscr{Z}_{A}|| x_{1} / x_{2} \mid=1\right\} \text {. }
$$


The interval of $T$-stability is given by:

$$
I_{A}(x)_{\mathbb{P}^{1}}:=\overline{\left\{t^{-1} \cdot 0 \mid t \cdot x \in \mathscr{Z}_{0, A}, \quad t \in T\right\} .}
$$

Here $x \in \mathscr{Z}_{A}$ and $T$ denotes the analytification of $T \otimes K$. bility

Define: $\mathscr{Z}:=\bigcap_{g \in S U_{2}(L)} g\left(\mathscr{Z}_{A}\right)$ and for $x \in \mathscr{Z}$ take as interval of $S U_{2}(L)$ semista-

$$
I(x)_{\mathbb{P}^{1}}:=\left\{z \in B_{2} \mid \forall(A \ni z) \quad z \in I_{A}(x)_{\mathbb{P}^{1}}\right\} .
$$

Note that $\mathscr{Z}=\tilde{\Omega}_{1}:=\mathbb{P}_{L}^{1}-\left\{x \mid x_{1} / x_{2}=c \cdot \gamma, c \in K\right.$ or $\left.x_{2}=0\right\}$. Here $\gamma \in L$ is an element such that $\gamma+\bar{\gamma}=0, \gamma \neq 0$.

From $\left(\mathbb{P}^{1}\right)_{T}^{s}=\left(\mathbb{P}^{1}\right)_{T}^{s s}$ one directly concludes the following:

PROPOSITION 5.3. (1) For $x \in \mathscr{Z}_{A}$ the interval $I_{A}(x)_{\text {pI }}$ is a point.

(2) For $x \in \mathscr{Z}$ the interval $I(x)_{\mathbb{P}^{1}}$ is a point.

DEFINITION 5.4. Let $H \subset B$ be the $S U_{2}(L)$ building regarded as a subcomplex of the $S U_{3}(L)$ building $B$. Let $A \subset H$ be an apartment. We define the following spaces:

$$
\begin{aligned}
Y_{H}^{s s} & :=\bigcap_{g \in S U_{2}(L)} g\left(Y_{A}^{s s}\right) ; \\
Y_{H}^{s} & :=\bigcap_{g \in S U_{2}(L)} g\left(Y_{A}^{s}\right) .
\end{aligned}
$$

For $x \in Y_{H}^{s s}$ one can now define the interval of $S U_{2}(L)$ semistability by:

$$
I_{H}(x):=\left\{z \in H \mid \forall(A \ni z \wedge A \subset H) \quad z \in I_{A}(x)\right\} .
$$

We take $\varphi: \mathbb{P}_{L^{0}}^{2}-\{(1,0,0)\} \rightarrow \mathbb{P}_{L^{0}}^{1}, \varphi\left(x_{0}, x_{1}, x_{2}\right)=\left(x_{1}, x_{2}\right)$ as before. We will also denote the map $\varphi \otimes L: \mathbb{P}_{L}^{2}-\{(1,0,0)\} \rightarrow \mathbb{P}_{L}^{1}$ by $\varphi$.

PROPOSITION 5.5. $Y_{H}^{s}=\varphi^{-1}\left(\tilde{\Omega}_{1}\right)=\varphi^{-1}(\mathscr{Z})$.

PROOF. One easily sees that if $A \subset H$, then $Y_{A}^{s}=\varphi^{-1}\left(\mathscr{Z}_{A}\right)$. Now the proposition follows from the definitions of $Y_{H}^{s}$ and $\mathscr{Z}$ and the fact that $\varphi$ is $S U_{2}(L)$-equivariant.

PROPOSITION 5.6. For $x \in Y_{A}^{s}$ one has $v_{A}(x)=I_{A}(\varphi(x))_{\mathbb{P}^{1}}$ and

$$
I_{A}(x)=\left\{z \in A \mid \operatorname{dist}\left(z, I_{A}(\varphi(x))_{\mathbb{P}^{1}}\right) \leq \max \left(0, v\left(x_{1} x_{2} / x_{0}^{2}\right)\right)\right\} .
$$

PROOF. Since $s \cdot v_{A}(x)=v_{A}(s \cdot x)$ and $\varphi$ is $S$-equivariant it is sufficient to proof it for $x$ with $\left|x_{1}\right|=\left|x_{2}\right|$. Then $v_{A}(x)=0$ and clearly $\varphi(x) \in \mathscr{Z}_{0, A}$. Hence $v_{A}(x)=$ $I_{A}(\varphi(x))_{\mathrm{pl}}=0$ in this case.

Now the second statement follows immediately from Corollary 2.11 . 
DEFINITION 5.7. For $y \in B$ and $r \geq 0$ define

$$
C(y, r):=\{z \in B \mid \operatorname{dist}(y, z) \leq r\} .
$$

Proposition 5.8. Let $x \in Y_{H}^{s s}$ and let $0 \in A$ be the usual vertex. Then

$$
C(0, r) \cap A \subset I_{H}(x) \text { implies } \quad C(0, r) \cap H \subset I_{H}(x) .
$$

PROOF. If $r=0$ the statement is trivial, so we assume $r>0$. Since $C(0, r) \cap A \subset$ $I_{A}(x)$ we have $v\left(x_{1} x_{2} / x_{0}^{2}\right) \geq r>0$. The fact that 0 is in the centre of $C(0, r)$ actually gives us $v\left(x_{i} / x_{0}\right) \geq r / 2>0, i=1,2$.

If $h \in P_{0} \cap S U_{2}(L)$, then $v\left(h^{*} x_{i}(x) / x_{0}\right) \geq \min \left(v\left(x_{1} / x_{0}\right), v\left(x_{2} / x_{0}\right)\right) \geq r / 2$, since $x \in Y_{0, A}^{s s}$. Hence $C(0, r) \cap h A \subset I_{h A}(x)$. Since $0 \in I_{H}(x)$ and $0 \in h A$, we use proposition 4.9 (2) to obtain $I_{h A}(x) \subset I_{H}(x)$ for all $h \in P_{0} \cap S U_{2}(L)$. Therefore $C(0, r) \cap H \subset I_{H}(x)$.

Definition 5.9. For $x \in Y_{H}^{s}$, we put $v_{H}(x):=I(\varphi(x))_{\text {pı }}$. We say $A$ determines $I_{H}(x)$ if:

(1) $\forall\left(A^{\prime} \subset H\right) \quad I_{A}(x) \subset A^{\prime}$ implies $I_{A^{\prime}}(x)=I_{A}(x)$.

(2) $x \in Y_{H}^{s}$ implies $v_{H}(x) \in A$.

$x \in Y_{H}^{s s}-Y_{H}^{s}$ implies $I_{A}(x)$ is not bounded.

THEOREM 5.10. Let $x \in Y_{H}^{s s}$ and suppose A determines $I_{H}(x)$. Then:

(1) $x \in Y_{H}^{s}$ implies $I_{H}(x)=C\left(v_{H}(x), \max \left(0, v\left(x_{1} x_{2} / x_{0}^{2}\right)\right)\right) \cap H$.

(2) $x=(1,0,0)$ implies $I_{H}(x)=H$.

(3) $x \in Y_{H}^{s s}-Y_{H}^{s}, x \neq(1,0,0)$ implies $I_{H}(x)=\bigcup_{g \in U_{2 \alpha}} g\left(I_{A}(x)\right)$. Here $U_{2 \alpha} \subset$ $S U_{2}(L)$ stabilises the limit point of the half-apartment containing $I_{A}(x)$.

PROOF. (1) Since $I_{A}(\varphi(x))_{\mathbb{P l}}=I(\varphi(x))_{\mathbb{p}^{1}}$ for all $A$ containing $I(\varphi(x))_{\mathbb{p} \text {, }}$, we have $v_{A}(x)=v_{H}(x)$ for all $A \subset H$ such that $v_{H}(x) \in A$. Furthermore, $\left|h^{*} x_{i} / x_{i}(x)\right|=1$, $i=1,2$ for all $h \in P_{\sigma} \cap S U_{2}(L)$. Here $v_{H}(x) \in \sigma \in A$.

Hence $I_{h A}(x)=C\left(v_{H}(x), \max \left(0, v\left(x_{1} x_{2} / x_{0}^{2}\right)\right)\right) \cap h A$ for all $h \in P_{\sigma} \cap S U_{2}(L)$. Furthermore $v_{H}(x) \in h A$ implies $I_{h A}(x) \subset I_{H}(x)$. Now it is clear that $I_{H}(x)=$ $H \cap C\left(v_{H}(x), \max \left(0, v\left(x_{1} x_{2} / x_{0}^{2}\right)\right)\right)$.

(2) If $x=(1,0,0)$ then $I_{A}(x)=A$. Since $x$ is a fixed point for the action of $S U_{2}(L)$, we easily conclude $I_{H}(x)=H$.

(3) Take $x \in Y^{s s}-Y^{s}$ such that $I_{A}(x)$ is a half-apartment and $A$ determines $I_{H}(x)$. Let $U_{2 \alpha}$ be the additive group in $S U_{2}(L) \subset S U_{3}(L)$ stabilising the limit point of $I_{A}(x)$. Let $g \in U_{2 \alpha}$. Then $A \cap g A$ is again a half-apartment. Clearly $I_{A}(x) \cap g A \subset I_{g A}(x)$. Let $y$ be the extremal point of $I_{A}(x) \subset A$. We may assume that the vertex $0 \in A \cap g A$ 
and $0 \in I_{A}(x)$. If $y \in g A$ then $I_{g A}(x)=I_{A}(x)$, since $A$ determines $I_{H}(x)$. So let us assume that $y \notin g A$.

If $y \notin g A$ then $C(0, \operatorname{dist}(0, y)) \cap A \subset I_{H}(x)$. Hence $C(0, \operatorname{dist}(0, y)) \cap H \subset I_{H}(x)$ by Proposition 5.8. In particular $g I_{A}(x) \subset I_{g A}(x)$. From the assumption that $\mathrm{A}$ determines $I_{H}(x)$ one easily concludes $I_{g A}(x)=g\left(I_{A}(x)\right)$. Clearly $I_{g A}(x) \subset I_{H}(x)$. Now statement (3) follows.

\section{The intervals of $S U_{3}(L)$-semistability}

6.1. Let $x \in Y^{s s}$. The interval of $S U_{3}(L)$ semistability $I(x)$ is convex. In particular one can find an apartment $A \subset B$ satisfying the following conditions:

(1) $\forall A^{\prime} \subset B \quad I_{A}(x) \subset A^{\prime}$ implies $I_{A^{\prime}}(x)=I_{A}(x)$

(2) $x \in Y^{s}$ implies $\left|I_{A}(x)\right|=\max \left\{\left|I_{A^{\prime}}(x)\right| \mid I_{A^{\prime}}(x) \subset I(x)\right\}$

$x \in Y^{s s}-Y^{s}$ implies $I_{A}(x)$ is not bounded.

If $A$ satisfies these conditions we say that $A$ determines $I(x)$. One easily sees that $I_{A}(x) \subset I(x)$ if $A$ determines $I(x)$.

The apartment $A$ is contained in a unique $S U_{2}(L)$ sub-building $H \subset B$. From the definitions it follows immediately that $A$ determines $I_{H}(x)$. In fact one has:

THEOREM 6.2. Let $x \in Y^{s s}$ and assume that $A \subset B$ determines I $(x)$. If $H$ is the $S U_{2}(L)$ sub-building that contains $A$ then $I(x)=I_{H}(x) \subset H \subset B$.

PROOF. From $I_{A}(x) \subset I(x)$ we conclude $1=r_{A, A}(x)=r(x)$. If $\sigma \in A$ with $\sigma \cap I_{A}(x) \neq \emptyset$, then $r_{h A, A}(x)=r(x)=1$ for all $h \in P_{\sigma}$. Hence $I_{h A}(x) \subset I(x)$. Taking the union of the $I_{h A}(x)$ for all $h \in P_{\sigma} \cap S U_{2}(L)$, for all $\sigma \in A$ such that $\sigma \cap I_{A}(x) \neq \emptyset$, we obtain $I_{H}(x)$. So $I_{H}(x) \subset I(x)$. Doing the same for all $h \in P_{\sigma}, \sigma$ as above we find $I_{H}(x)=I(x) \cap H$.

Suppose $I(x) \cap H \neq I(x)$. Then there exists a vertex $v$ and an apartment $A^{\prime}$ such that $A^{\prime} \cap H=\{v\}(\operatorname{char}(\bar{K}) \neq 2), v \in I(x) \cap H$ with $I_{A^{\prime}}(x) \neq\{v\}$. The embedding of $H \subset B$ is such that there exists $A^{\prime} \subset B$ such that $A^{\prime} \cap H=\{v\}$ only if $v$ is of type 0 . So it is sufficient to treat the case $v=0 \in A \subset H$.

Firstly we assume that 0 is an extremal point of $I_{H}(x)$ considered as a subset of $H$. If $I_{A^{\prime}}(x) \neq\{0\}$ there exists an apartment $A^{\prime \prime}$ such that $A^{\prime \prime} \supset I_{A}(x)$ and $I_{A^{\prime \prime}}(x) \cap I_{A^{\prime}}(x) \neq\{0\}$. Hence $I_{A}(x) \neq I_{A^{\prime \prime}}(x)$. This cannot be, since we assumed that $A$ determined $I(x)$.

Now we assume that 0 is not an extremal point of $I_{H}(x)$ considered as a subset of $H$. Now $A^{\prime}=h A$ with $h \in P_{0}$. We may assume $h=u_{\alpha}(a, b) u_{-\alpha}(c, d)$ with $|a|=|c|=1$. Since $h A \cap A=\{0\}$ we also need to assume $|c b+a|=1$ (this 
implies $\left|a-\bar{c}^{-1}\right|=\left|b+(c \bar{c})^{-1}\right|=1$.) Explicit calculations for $h$ as above show that $I_{h A}(x)=\{0\}$. Hence $I(x) \subset H$ and the theorem follows.

REMARK 6.3. If $A$ and $A^{\prime}$ are two apartments that determine $I(x)$, then the $S U_{2}(L)$ buildings $H$ and $H^{\prime}$ containing $A$ and $A^{\prime}$, respectively, might be different. However we still have: $I_{H}(x)=I_{H^{\prime}}(x)$. In particular, $I_{H}(x) \subset H \cap H^{\prime}$.

DEFINITION 6.4. Let $x \in Y^{s}$ and suppose $A$ determines $I(x)$. Then we define $v_{B}(x):=v_{A}(x)$. Note that this does not depend on the choice of the apartment $A$ that determines $I(x)$.

In the next proposition we show how $I_{A}(x)$ is related to $I(x)$ for any apartment $A$ in the building $B$.

Proposition 6.5. Let $A \subset B$ be an apartment and $x \in Y^{s s}$. Then

(1) $I_{A}(x)=I(x) \cap A$, if $A \cap I(x) \neq \emptyset$.

(2) $I_{A}(x)$ is the vertex in $A$ closest to $I(x)$, if $A \cap I(x)=\emptyset$.

PROOF. The first statement is a direct consequence of Proposition 4.9(2). So we only have to prove the second statement.

Let $A \subset B$ be an apartment such that $A \cap I(x)=\emptyset$. Let $\sigma \in A$ be a chamber such that $I_{A}(x) \cap \sigma \neq \emptyset$. There exists $g \in P_{\sigma}$ such that $g A \cap I(x) \neq \emptyset$. Hence $I_{g A}(x)=$ $I(x) \cap g A$, according to statement (1). Furthermore it follows from Proposition 4.6(a) that $r_{g A, A}(x)<1$.

Clearly we can choose $g$ in either $P_{\sigma} \cap U_{\alpha}$ or $P_{\sigma} \cap U_{-\alpha}$. We will only treat the case where $g \in P_{\sigma} \cap U_{\alpha}$, since the other case is similar. Without loss of generality we may assume that $A$ is our standard apartment and that $\sigma$ is the chamber corresponding to the modules $\left[M_{0}\right]$ and $\left[M_{-1}\right]$. Hence $g=u_{\alpha}(a, b)$ with $|a|,|b| \leq 1$.

We will firstly prove that $I_{A}(x)$ consists of a single point. Let us assume that $I_{A}(x)$ is not a point. Then $I_{A}(x) \cap \sigma=\left[2 v\left(x_{0} / x_{2}\right), 2 v\left(x_{1} / x_{0}\right)\right]_{A} \cap[-1,0]_{A} \neq \emptyset$. In particular $v\left(x_{0} / x_{2}\right) \leq 0$. Hence $\left|x_{2} / x_{0}\right| \leq 1$. Furthermore $\left|x_{1} x_{2} / x_{0}^{2}\right|<1$. Since $r_{g A, A}(x)<1$, we must have $\left|g^{*} x_{0} / x_{0}(x)\right|=\left|\left(x_{0}-a x_{2}\right) / x_{0}\right|<1$. Since $|a| \leq 1$ and $\left|x_{2} / x_{0}\right| \leq 1$, we must have $|a|=1$ and $\left|x_{2} / x_{0}\right|=1$. Since $\left|x_{1} x_{2} / x_{0}^{2}\right|<1$, we also have $\left|x_{1} / x_{0}\right|<1$.

We have $g^{*} x_{1}=x_{1}+2 \bar{a} x_{0}-b x_{2}$. From $\left|\left(x_{0}-a x_{2}\right) / x_{0}\right|<1$ and $\left|x_{0} / x_{2}\right|=1$ it follows that $\left|2 \bar{a} x_{0}-b x_{2} / x_{0}\right|=1$. Furthermore $\left|g^{*} x_{1} / x_{0}(x)\right|=\left|g^{*} x_{2} / x_{0}(x)\right|=1$ and $\left|g^{*} x_{0} / x_{0}(x)\right|<1$. So $I_{g A}(x)$ is the vertex 0 . This contradicts our assumption that $A \cap I(x)=\emptyset$. Therefore $I_{A}(x)$ has to be a point.

Now let us assume that $I_{A}(x)$ consists of a single point. Our assumptions are such that we must show that $I_{A}(x)=\{0\}$, since this is clearly the vertex in $\sigma$ closest to 
$I(x)$. We must furthermore show that 0 is the vertex in $A$ closest to $I(x)$. Since $r_{g A, A}(x)<1$ and $g^{*} x_{2}=x_{2}$, we must have $\left|g^{*} x_{1} / x_{1}(x)\right|<1$.

If $\left|x_{0}^{2} / x_{1} x_{2}(x)\right|<1$, then $|b|=1$ and $\left|x_{2} / x_{1}(x)\right|=1$. Hence $I_{A}(x)=\{0\}$. Since $|b|=1$, it is clear that 0 is the vertex in $A$ closest to $I(x)$.

If $\left|x_{0}^{2} / x_{1} x_{2}(x)\right|=1$ then $\left|g^{*} x_{0} / x_{0}(x)\right|<1$. Hence $\left|x_{1} / x_{0}(x)\right|=\left|x_{2} / x_{0}(x)\right|=1$. Again we conclude that $I_{A}(x)=\{0\}$, and furthermore $|a|=1$. Hence again 0 is the vertex in $A$ closest to $I(x)$. This concludes the proof of the proposition.

\section{A pure affinoid covering of $Y^{s}$}

7.1. The description of $I(x)$ given above, enables us to give a pure affinoid covering of $Y^{s}$. The affinoids used will be nice open affinoid subspaces of the affinoids $Y\left\{\rho_{1}, \rho_{2}, \sigma\right\}_{A}$. The components of the reduction of $Y^{s}$ with respect to this pure affinoid covering $\mathscr{C}$ will be in 1-1 correspondance with certain convex subsets of the building.

DEFINITION 7.2. Let $A \subset B$ be an apartment. Let $H \subset B$ be the $S U_{2}(L)$ subbuilding determined by $A$.

An A-stable polyhedron $\Delta_{A} \subset A$ is the convex hull of two vertices of type 0 $\tau_{1}, \tau_{2} \in A$. We write $\Delta_{A}=\left[\tau_{1}, \tau_{2}\right]_{A}$.

Let $\Delta_{A}=\left[\tau_{1}, \tau_{2}\right]_{A}$ be an $A$-stable polyhedron and let $v_{A}\left(\Delta_{A}\right)$ denote the center of $\Delta_{A}$. Then $v_{A}\left(\Delta_{A}\right)$ is the unique point $z \in A$ such that $\operatorname{dist}\left(\tau_{1}, z\right)=\operatorname{dist}\left(\tau_{2}, z\right)$. Suppose $v_{A}\left(\Delta_{A}\right) \in \sigma \in A$. We call $\Delta=\bigcup_{g \in P_{\sigma} \cap s U_{2}(L)} g\left(\Delta_{A}\right)$ a stable polyhedron. Here $S U_{2}(L)$ is the group acting on $H \subset B$. We write $\Delta=\left[\tau_{1}, \tau_{2}\right]$. Note that $\Delta \subset H$.

The stable polyhedron $\Delta$ is uniquely determined by $\tau_{1}$ and $\tau_{2}$. If we take another apartment $A^{\prime} \ni \tau_{1}, \tau_{2}$, the corresponding $S U_{2}(L)$ sub-building $H^{\prime} \subset B$ contains $\Delta$, that is, $\Delta \subset H \cap H^{\prime}$.

The center of $\Delta$ is denoted by $v_{B}(\Delta)$.

We say $A$ determines $\Delta$ if $\Delta=\left[\tau_{1}, \tau_{2}\right]$ with $\tau_{i} \in A$. Note that $v_{B}(\Delta) \in A$ if $A$ determines $\Delta$.

DeFinition 7.3. For $g \in S U_{3}(L)$ we put $Y\left\{g\left(\rho_{1}\right), g\left(\rho_{2}\right), g(\sigma)\right\}_{g A}:=g\left(Y\left\{\rho_{1}, \rho_{2}, \sigma\right\}_{A}\right)$. Now define:

$$
Y\left\{\rho_{1}, \rho_{2}, \sigma\right\}:=\left\{x \in Y\left\{\rho_{1}, \rho_{2}, \sigma\right\}_{A} \mid A \text { determines } I(x)\right\} .
$$

If $\rho_{1}=\rho_{2}=: \rho$ and $\sigma \subset \rho$, then:

$$
Y\{\rho, \rho, \sigma\}=\left\{x \in Y^{s} \mid I(x) \subset \rho, v_{B}(x) \in \sigma\right\} \supset Y_{\sigma}^{s} .
$$

If $\rho_{1} \neq \rho_{2}$ then we write $\rho_{i}=\left[\tau_{1}^{i}, \tau_{2}^{i}\right]$ with $\operatorname{dist}\left(\tau_{1}^{1}, \tau_{1}^{2}\right)=\operatorname{dist}\left(\tau_{2}^{1}, \tau_{2}^{2}\right)-4$. Then 


$$
Y\left\{\rho_{1}, \rho_{2}, \sigma\right\}=\left\{x \in Y^{s} \mid\left[\tau_{1}^{1}, \tau_{1}^{2}\right] \subset I(x) \subset\left[\tau_{2}^{1}, \tau_{2}^{2}\right], \quad v_{B}(x) \in \sigma\right\} .
$$

We denote the covering $\left\{Y\left\{\rho_{1}, \rho_{2}, \sigma\right\} \mid \rho_{i}, \sigma \in B\right\}$ by $\mathscr{C}$.

Note that the affinoid covering given here differs from the one given in [9]. There are some mistakes in [9]: the covering given there is wrong. (Luckily [9] is not easily obtained outside Japan.)

PROPOSITION 7.4. Let $R: Y\left\{\rho_{1}, \rho_{2}, \sigma\right\}_{A} \rightarrow R\left(Y\left\{\rho_{1}, \rho_{2}, \sigma\right\}_{A}\right)$ denote the canonical reduction map. Then there exists an open affine set $V \subset R\left(Y\left\{\rho_{1}, \rho_{2}, \sigma\right\}_{A}\right)$ such that $R^{-1}(V)=Y\left\{\rho_{1}, \rho_{2}, \sigma\right\}$.

In particular $Y\left\{\rho_{1}, \rho_{2}, \sigma\right\}$ is affinoid and its canonical reduction is $V$.

PROOF. We prove the proposition by giving an explicit description of $Y\left\{\rho_{1}, \rho_{2}, \sigma\right\} \subset$ $Y\left\{\rho_{1}, \rho_{2}, \sigma\right\}_{A}$ as an open subset. For a convex subset $\Delta \subset B$ we denote by $P(\Delta)^{-}$ the (not pointwise) stabiliser of $\Delta$ in $S U_{3}(L)$.

Firstly we treat the case $\rho_{1}=\rho_{2}$. We take:

$$
W:=\left\{x \in Y\left\{\rho_{1}, \rho_{1}, \sigma\right\}_{A}\left|\forall\left(g \in P_{\sigma}\right)\right| g^{*} x_{i} / x_{i}(x) \mid=1, i=1,2\right\}
$$

Firstly we will show that $W=Y\left\{\rho_{1}, \rho_{1}, \sigma\right\}$. If $x \in Y\left\{\rho_{1}, \rho_{1}, \sigma\right\}_{A}$ then $I_{A}(x) \subset$ $I(x)$ or $r_{g A, A}(x)<1$. Hence $\left|g^{*} x_{1} g^{*} x_{2} / x_{1} x_{2}(x)\right| \leq 1$, since $\left|I_{g A}(x)\right| \geq\left|I_{A}(x)\right|$ or $r_{g A, A}(x)<1$. If $x \in Y\left\{\rho_{1}, \rho_{1}, \sigma\right\}$ then $\left|g^{*} x_{1} g^{*} x_{2} / x_{1} x_{2}(x)\right|=1$ for all $g \in P_{\sigma}$, since $I_{g A}(x)=g\left(I_{A}(x)\right)$. Furthermore for each $g \in P_{\sigma}$ there are apartments $A^{\prime}$ and $A^{\prime \prime}$ that correspond to the coordinates $g^{*} x_{1}, x_{2}$ and $g^{*} x_{2}, x_{1}$, respectively. Therefore if $x$ is in $Y\left\{\rho_{1}, \rho_{1}, \sigma\right\}_{A}$ then for all $g \in P_{\sigma}\left|g^{*} x_{i} / x_{i}(x)\right| \leq 1, i=1,2$, and furthermore $W=$ $Y\left\{\rho_{1}, \rho_{1}, \sigma\right\}$. Now it is clear that $R(W)$ is an open affine subset of $R\left(Y\left\{\rho_{1}, \rho_{1}, \sigma\right\}_{A}\right)$. Clearly $W=R^{-1}(R(W))$, so the statement is true if $\rho_{1}=\rho_{2}$.

Suppose $\rho_{1} \neq \rho_{2}$. As before we write $\rho_{i}=\left[\tau_{1}^{i}, \tau_{2}^{i}\right]$ with $\operatorname{dist}\left(\tau_{1}^{1}, \tau_{1}^{2}\right)+4=$ $\operatorname{dist}\left(\tau_{2}^{1}, \tau_{2}^{2}\right)$. The set of extremal vertices of the stable polyhedron $\left[\tau_{1}^{1}, \tau_{1}^{2}\right]$ will be denoted by $\mathscr{V}:=\left\{V_{1} \cdots V_{S}\right\}$. Let $A_{i} \subset H$ denote an apartment containing $V_{i}$. Now the proof is similar to in the case $\rho_{1}=\rho_{2}$ using the following subsets of $Y\left\{\rho_{1}, \rho_{2}, \sigma\right\}_{A}$ :

$$
\begin{aligned}
Z_{0} & :=Y\left\{\rho_{1}, \rho_{2}, \sigma\right\}_{A} \\
Z_{1} & :=\left\{x \in Z_{0} \mid \quad v_{H}(x)=v_{A}(x)\right\} \\
& =\left\{x \in Z_{0}\left|\forall\left(g \in P_{\sigma} \cap S U_{2}(L)\right)\right| g^{*} x_{i} / x_{i}(x) \mid=1, \quad i=1,2\right\} \\
Z_{2} & :=\left\{x \in Z_{1} \mid \forall\left(H^{\prime} \supset\left[\tau_{2}^{1}, \tau_{2}^{2}\right]\right) v_{H^{\prime}}(x)=v_{H}(x)\right\} \\
& =\left\{x \in Z_{1}\left|\forall\left(g \in P\left(\left[\tau_{2}^{1}, \tau_{2}^{2}\right]\right)^{-} \cap P_{\sigma}\right)\right| g^{*} x_{i} / x_{i}(x) \mid=1, \quad i=0,1,2\right\} \\
Z_{3} & :=\left\{x \in Z_{2} \mid I(x)=I_{H}(x)\right\} \\
& =\left\{x \in Z_{2} \mid \forall(V \in \mathscr{V}) \forall\left(g \in P_{V}\right) g A_{j} \cap H=\{V\} \text { implies }\left|g^{*} x_{1} g^{*} x_{2} / x_{0}^{2}(x)\right|=1\right\} \\
& =Y\left\{\rho_{1}, \rho_{2}, \sigma\right\} .
\end{aligned}
$$


Now $R\left(Z_{3}\right) \subset R\left(Z_{2}\right) \subset R\left(Z_{1}\right) \subset R\left(Z_{0}\right)$. Furthermore $R\left(Z_{i}\right) \subset R\left(Z_{i-1}\right), i=1,2,3$ is an open and affine subset and $R^{-1}\left(R\left(Z_{i}\right)\right)=Z_{i}$. This proves the proposition.

THEOREM 7.5. (1) $\bigcup_{\rho_{1}, \rho_{2}, \sigma \in B} Y\left\{\rho_{1}, \rho_{2}, \sigma\right\}=Y^{s}$.

(2) The covering $\mathscr{C}:=\left\{Y\left\{\rho_{1}, \rho_{2}, \sigma\right\} \mid \rho_{1}, \rho_{2}, \sigma \in B\right\}$ is pure.

(3) The reduction of $Y^{s}$ with respect to the covering $\mathscr{C}$ consists of proper components.

(4) The components of the reduction are in 1-1 correspondance with the stable polyhedra.

Proof. The first statement is evident from the construction. The second statement follows from Proposition 7.4 and the fact that the covering $\mathscr{C}_{A}$ is pure (Proposition 3.2(3)). Statement (3) follows from statement (1) as in [4, proof of 3.6 part 5]. The last statement follows from Propositions 3.2(5) and 7.4.

\section{The reduction of $Y^{s}$}

8.1. In this section we describe the reduction of $Y^{s}$. We firstly determine the reduction of $Y_{A}^{s}$ with respect to the pure affinoid covering $\mathscr{C}_{A}$. Then we use the stabilisers of the components of the reduction to determine the reduction of $Y^{s}$ with respect to $\mathscr{C}$.

DEFINITION 8.2. For a stable polyhedron $\Delta \subset B$ such that the apartment $A \subset B$ determines $\Delta$ we define:

$$
\begin{aligned}
Y(\Delta)_{A} & :=\left\{x \in Y_{A}^{s} \mid I_{A}(x)=\Delta \cap A\right\} \\
Y(\Delta) & :=\left\{x \in Y^{s} \mid I(x)=\Delta\right\} \\
P(\Delta)^{-} & :=\left\{g \in S U_{3}(L) \mid g(\Delta)=\Delta\right\}
\end{aligned}
$$

The canonical reduction of $Y(\Delta)_{A}, Y(\Delta)$ etcetera will be denoted by $\overline{Y(\Delta)_{A}}, \overline{Y(\Delta)}$, and so on.

Note that if $\Delta \cap A$ is the convex hull of the vertices $\tau_{1}$ and $\tau_{2}$, then $Y(\Delta)_{A}=$ $Y\left\{\tau_{1}, \tau_{2}, \sigma\right\}_{A}$. Here the chamber $\sigma$ is choosen in such a way that $v_{A}(\Delta \cap A) \in \sigma$.

The following proposition is rather obvious and we omit the proof.

PROPOSITION 8.3. (1) $Y(\Delta)_{A} \subset Y\left\{\rho_{1}, \rho_{2}, \sigma\right\}_{A}$ if and only if $\Delta \cap \rho_{1} \neq \emptyset, \Delta \cap \rho_{2} \neq$ $\emptyset v_{B}(\Delta) \in \sigma$ and $\Delta \cap A \subset$ convex hull of $\left\{\rho_{1}, \rho_{2}\right\}$.

(2) $Y(\Delta)_{A}=\cap Y\left\{\rho_{1}, \rho_{2}, \sigma\right\}_{A}$ with $\Delta \cap \rho_{1} \neq \emptyset, \Delta \cap \rho_{2} \neq \emptyset$ and $v_{B}(\Delta) \in \sigma$ and $\Delta \cap A \subset$ convex hull of $\left\{\rho_{1}, \rho_{2}\right\}$. 
(3) $Y(\Delta)_{A} \subset Y\left\{\rho_{1}, \rho_{2}, \sigma\right\}_{A}$ implies $\overline{Y(\Delta)_{A}}$ is in the component of $\overline{Y\left\{\rho_{1}, \rho_{2}, \sigma\right\}_{A}}$ corresponding to $\Delta_{A}$. Furthermore $\overline{Y(\Delta)_{A}}$ is open and affine in $\overline{Y\left\{\rho_{1}, \rho_{2}, \sigma\right\}_{A}}$ and $Y(\Delta)_{A}=R^{-1}\left(\overline{Y(\Delta)_{A}}\right)$.

(4) $Y(\Delta)=\bigcap_{g \in P(\Delta)^{-}} g\left(Y(\Delta)_{A}\right)=\bigcap_{g \in P(\Delta)^{-}} Y(\Delta)_{g A}$.

(5) $Y(\Delta)=\left\{x \in Y(\Delta)_{A}|| g^{*} x_{i} / x_{i}(x) \mid=1, i=1,2 \forall g \in P(\Delta)^{-}\right\}$.

(6) $\overline{Y(\Delta)} \subset \overline{Y(\Delta)_{A}}$ is open and affine and $R^{-1}(\overline{Y(\Delta)})=Y(\Delta)$, where $R: Y(\Delta)_{A} \rightarrow$ $\overline{Y(\Delta)_{A}}$ is the canonical reduction map.

DEFINITION 8.4. Before describing the reduction of $Y_{A}^{s}$ we need some definitions. Let $A$ be the standard apartment and let the vertices correspond with the integers as before.

For an $A$-stable polyhedron $\Delta_{A}=[2 n, 2 m]_{A}$, the centre $v_{A}(\Delta)$ and its length $\left|\Delta_{A}\right|$ are given by $v_{A}\left(\Delta_{A}\right)=(n+m), \quad\left|\Delta_{A}\right|=|2 m-2 n|$.

We put a simplicial structure on the set of $A$-stable polyhedra. The collection of simplices will be denoted by $\mathscr{P}_{A}$. The elements of $\mathscr{P}_{A}$ are the non-empty subsets of the following sets:

$$
\left\{\Delta_{A}^{1}, \Delta_{A}^{2}, \Delta_{A}^{3}\left|\Delta_{A}^{1} \subset \Delta_{A}^{2} \subset \Delta_{A}^{3}, v_{A}\left(\Delta_{A}^{1}\right)=v_{A}\left(\Delta_{A}^{2}\right),\right| \Delta_{A}^{3}|=| \Delta_{A}^{2}|+2=| \Delta_{A}^{1} \mid+4\right\}
$$

Note that the triangles in the picture of $\mathscr{C}_{A}$ correspond to the maximal simplices.

Proposition 8.5. Let $\Delta_{A}=[2 i, 2 j]_{A}$ with $i \leq j$. The component $X\left(\Delta_{A}\right)$ of the reduction of $Y_{A}^{s}$ corresponding to $\Delta_{A}$ is a $\mathbb{P}_{\bar{L}}^{2}$ with a point blown up for the $\Delta_{A}^{\prime}$ such that $\left\{\Delta_{A}, \Delta_{A}^{\prime}\right\} \in \mathscr{P}_{A}$ and $\left|\Delta_{A}\right|=\left|\Delta_{A}^{\prime}\right|+4$ or $\left|\Delta_{A}^{\prime}\right|=\left|\Delta_{A}\right|-2$.

The intersections with the other components of the reduction are:

(1) $X\left(\Delta_{A}\right) \cap X\left(\Delta_{A}^{\prime}\right)$ is an exceptional line in $X\left(\Delta_{A}\right)$ if:

$$
\left\{\Delta_{A}, \Delta_{A}^{\prime}\right\} \in \mathscr{P}_{A} \text { and }\left|\Delta_{A}^{\prime}\right|=\left|\Delta_{A}\right|+4 \text { or }\left|\Delta_{A}^{\prime}\right|=\left|\Delta_{A}\right|-2
$$

(2) $X\left(\Delta_{A}\right) \cap X\left(\Delta_{A}^{\prime}\right)$ is an ordinary line in $X\left(\Delta_{A}\right)$ if:

$$
\left\{\Delta_{A}, \Delta_{A}^{\prime}\right\} \in \mathscr{P}_{A} \text { and }\left|\Delta_{A}^{\prime}\right|=\left|\Delta_{A}\right|-4 \text { or }\left|\Delta_{A}^{\prime}\right|=\left|\Delta_{A}\right|+2
$$

(3) $X\left(\Delta_{A}\right) \cap X\left(\Delta_{A}^{\prime}\right) \cap X\left(\Delta_{A}^{\prime \prime}\right)$ is a point if and only if $\left\{\Delta_{A}, \Delta_{A}^{\prime}, \Delta_{A}^{\prime \prime}\right\} \in \mathscr{P}_{A}$.

(4) $X\left(\Delta_{A}\right) \cap X\left(\Delta_{A}^{\prime}\right)=\emptyset$ if $\left\{\Delta_{A}, \Delta_{A}^{\prime},\right\} \notin \mathscr{P}_{A}$.

ProOF. We firstly treat the case $\Delta_{A}=[0,0]_{A}$. One calculates $X\left(\Delta_{A}\right)$ using torus embeddings (see [6]). The picture of the covering $\mathscr{C}_{A}$ more or less directly gives $\left(X\left(\Delta_{A}\right)\right.$.

The first picture (see below) shows that the affines of the reduction glue together in a $\mathbb{P}_{\bar{L}}^{2}$, corresponding to $\operatorname{Proj}\left(\bar{L}\left[\bar{x}_{0}, \bar{x}_{1}, \bar{x}_{2}\right]\right)$. The actual picture at $\Delta_{A}=[0,0]_{A}$ is a subdivision of this picture. This extra line gives a blow up of a point. In our case the 
point is given by $\bar{x}_{1} / x_{0}=\bar{x}_{2} / x_{0}=0$. Hence we find $X\left([0,0]_{A}\right)$ is a $\mathbb{P}_{\bar{L}}^{2}$ with a point blown up.
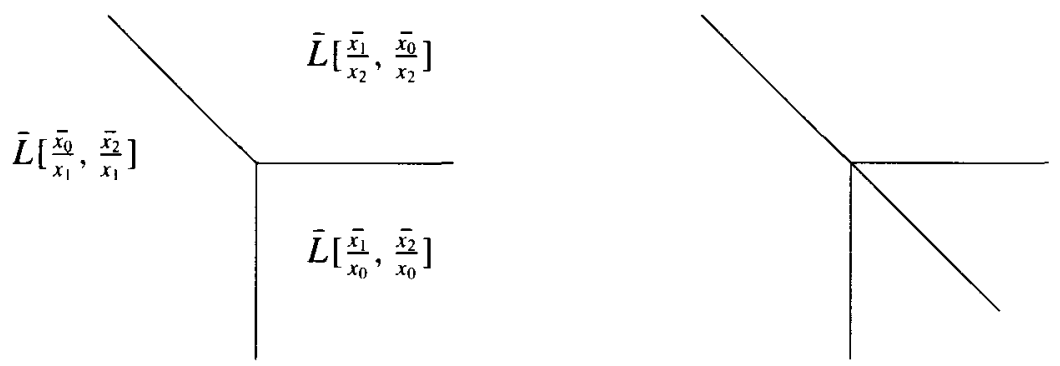

The line connecting $[0,0]_{A}$ with $[-2,2]_{A}$ in the picture (see 3.1$)$ gives the intersection $X\left([0,0]_{A}\right) \cap X\left([-2,2]_{A}\right)$. It corresponds to the exceptional line in $\mathbb{P}_{\bar{L}}^{2}$. Furthermore $X\left([0,2]_{A}\right) \cap X\left([0,0]_{A}\right)$ and $X\left([-2,0]_{A}\right) \cap X\left([0,0]_{A}\right)$ are ordinary lines in $X\left([0,0]_{A}\right)$.

The intersection $X\left([0,0]_{A}\right) \cap X\left(\Delta_{A}\right) \cap X\left(\Delta_{A}^{\prime}\right)$ is a point if there exists an affinoid $F$ in $\mathscr{C}_{A}$ such that $[0,0]_{A}, \Delta_{A}$ and $\Delta_{A}^{\prime}$ correspond to vertices of $F$ in the picture. Hence $\left\{[0,0], \Delta_{A}, \Delta_{A}^{\prime}\right\} \in \mathscr{P}_{A}$.

If $\left\{[0,0]_{A}, \Delta_{A}\right\} \notin \mathscr{P}_{A}$, then there does not exist an affinoid in the covering $\mathscr{C}_{A}$ having a component corresponding to both $A$-stable polyhedra. Hence the intersection of the corresponding components is empty.

From this one concludes that the proposition is true for $\Delta_{A}=[0,0]_{A}$. For the other $\Delta_{A}$ that are vertices $[2 n, 2 n]_{A}$ the situation is exactly the same.

If $\Delta_{A}$ is not a vertex of $A$ then the picture around the component $\Delta_{A}$ has two more lines in it. Hence the component $X\left(\Delta_{A}\right)$ is a $\mathbb{P}_{\bar{L}}^{2}$ with $(1,0,0),(0,1,0)$ and $(0,0,1)$ blown up. Now one proves the proposition in a similar vein as for $\Delta_{A}=[0,0]_{A}$. This concludes the proof.

DEFINITION 8.6. We take $\mathscr{C}_{A}^{\prime}:=\left\{Y\left\{\rho_{1}, \rho_{2}, \sigma\right\} \mid \rho_{1}, \rho_{2}, \sigma \in A\right\}$ and we denote by $X(\Delta)_{A}$ the component of the reduction with respect to this covering belonging to $\Delta_{A}$. Here $\Delta$ is a stable polyhedron such that $A$ determines $\Delta$. Hence $\Delta$ is uniquely determined by $\Delta_{A}=\Delta \cap A$.

Since $\overline{Y\left\{\rho_{1}, \rho_{2}, \sigma\right\}} \subset \overline{Y\left\{\rho_{1}, \rho_{2}, \sigma\right\}_{A}}$ is open and affine, $X(\Delta)_{A} \subset X(\Delta \cap A)$ is a Zariski open subset.

The action of $g \in P(\Delta)^{-}$on $Y(\Delta)$ induces an action $\bar{g}$ on the reduction $\overline{Y(\Delta)}$ of $Y(\Delta)$. We put $\overline{P(\Delta)}:=\left\{\bar{g} \mid g \in P(\Delta)^{-}\right\}$. The action of $\overline{P(\Delta)}$ on $\overline{Y(\Delta)}$ can be extended to the component $X(\Delta)$. Here $X(\Delta)$ is the component corresponding to $\Delta$ of the reduction of $Y^{s}$ with respect to $\mathscr{C}$. Clearly $\overline{Y(\Delta)} \subset X(\Delta)$. Furthermore one 
has $X(\Delta)_{A} \supset \overline{Y(\Delta)}$. In fact one has:

PROPOSITION 8.7. $X(\Delta)=\bigcup_{g \in P(\Delta)^{-}} X(\Delta)_{g A}=\bigcup_{\bar{g} \in \overline{P(\Delta)^{-}}} \bar{g}\left(X(\Delta)_{A}\right)$.

Proof. This is clear since $P(\Delta)^{-}$acts transitively on the apartments $A$ such that $A$ determines $\Delta$, that is, $|\Delta \cap A|$ is maximal.

Proposition 8.8. Let $\Delta$ be a stable polyhedron and let $A$ determine $\Delta$. Then $X(\Delta)_{A} \subset X(\Delta \cap A)$ is the open subset obtained by omitting the images of the lines $g^{*} x_{i}=0, i=1,2, g \in P(\Delta)^{-}$, that do not coincide with the images of $x_{1}=0$ or $x_{2}=0$ in $X(\Delta \cap A)$.

ProOF. This follows more or less directly from the description of $Y\left\{\rho_{1}, \rho_{2}, \sigma\right\} \subset$ $Y\left\{\rho_{1}, \rho_{2}, \sigma\right\}_{A}$ given in the proof of Proposition 7.4.

DEFINITION 8.9. For a stable polyhedron $\Delta$ we put $|\Delta|=\max _{A}|\Delta \cap A|$. Furthermore we put a simplicial structure on the set of stable polyhedra. The collection $\mathscr{P}$ of simplices has as its elements the non-empty subsets of the sets:

$\left\{\Delta_{1}, \Delta_{2}, \Delta_{3}|\exists(A \subset B)| \Delta_{i} \cap A|=| \Delta_{i} \mid \quad\right.$ and $\left.\quad\left\{\Delta_{1} \cap A, \Delta_{2} \cap A, \Delta_{3} \cap A\right\} \in \mathscr{P}_{A}\right\}$.

PROPOSITION 8.10. Let A determine the stable polyhedron $\Delta$. If $\Delta=[2 i, 2 j]$ with $i \leq j$ then the component of the reduction $X(\Delta)$ belonging to $\Delta$ consists of $a \mathbb{P}_{\bar{L}}^{2}$ with a point blown up for each $\Delta^{\prime}$ such that $\left\{\Delta, \Delta^{\prime}\right\} \in \mathscr{P}$ and $\left|\Delta^{\prime}\right|=|\Delta|+4$ or $\left|\Delta^{\prime}\right|=|\Delta|-2$.

The intersections with other components are as follows:

(1) $X(\Delta) \cap X\left(\Delta^{\prime}\right)$ is an exceptional line in $X(\Delta)$ if:

$$
\left\{\Delta, \Delta^{\prime}\right\} \in \mathscr{P} \text { and }\left|\Delta^{\prime}\right|=|\Delta|+4 \text { or }|\Delta|-2 .
$$

(2) $X(\Delta) \cap X\left(\Delta^{\prime}\right)$ is an ordinary line in $X(\Delta)$ if:

$$
\left\{\Delta, \Delta^{\prime}\right\} \in \mathscr{P} \text { and }\left|\Delta^{\prime}\right|=|\Delta|-4 \text { or }\left|\Delta^{\prime}\right|=|\Delta|+2 .
$$

(3) $X(\Delta) \cap X\left(\Delta^{\prime}\right) \cap X\left(\Delta^{\prime \prime}\right)$ is a point if and only if $\left\{\Delta, \Delta^{\prime}, \Delta^{\prime \prime}\right\} \in \mathscr{P}$.

(4) $X(\Delta) \cap X\left(\Delta^{\prime}\right)=\emptyset$ if $\left\{\Delta, \Delta^{\prime}\right\} \notin \mathscr{P}$.

PROOF. This follows directly from the previous propositions using the fact that $\overline{P(\Delta)^{-}}$acts linearly on the $\mathbb{P}_{\bar{L}}^{2}$. 
REMARK 8.11. One can embed $S U_{3}(L)$ into $S L_{3}(L)$. The maximal $K$-split torus $S(K) \cong L^{*}$ of $S U_{3}(L)$ is contained in a unique maximal $L$-split torus $T \subset S L_{3}(L)$, which again acts diagonally on $\mathbb{P}_{L}^{2}$ with respect to the coordinates $x_{0}, x_{1}, x_{2}$. Hence $S(K)$ determines a unique apartment $A$ in the building $B_{3}$ of $S L_{3}(L)$.

Let $\mathscr{H}:=\bigcup_{g \in S U_{3}(L)} g \cdot A \subset B_{3}$. Then $\mathscr{H}$ is a convex subcomplex of $B_{3}$. The vertices of $\mathscr{H}$ correspond 1-1 with the $S U_{3}(L)$-images of $\left[<e_{0}, \pi^{n} e_{1}, \pi^{m} e_{2}>\right.$ ], $n, m \in \mathbb{Z}$. The maximal simplices are triangles. The three equivalence classes $\left[\tilde{N}_{i}\right]$, $i=1,2,3$, correspond to a maximal simplex, if there exist representatives $N_{i} \in\left[\tilde{N}_{i}\right]$ such that $N_{1} \supset N_{2} \supset N_{3} \supset \pi N_{1}$.

Let $Y_{\mathscr{H}}:=\left\{z \in Y^{s} \mid \forall\left(g \in S U_{3}(L)\right) \quad g^{*} x_{0}(z) \neq 0\right\}$. Since $\mathscr{H} \subset B_{3}$ is convex, there exists a formal scheme for $Y_{\mathscr{H}}$ whose closed fibre consists of a proper component for each vertex of $\mathscr{H}$ (See [5]). These components are of the form $\mathbb{P}_{\bar{L}}^{2}$ with some points blown up.

One can associate to each stable polyhedron $\Delta$ a unique equivalence class $\left[M_{\Delta}\right]$ of $L^{0}$ modules as follows. Suppose $A$ determines $\Delta$. We can find an $x \in Y^{s}$ such that $I(x)=\Delta$. Then $n_{i}:=v\left(x_{i} / x_{0}\right) \in \mathbb{Z}$ for $i=1,2$. The integers $n_{i}$ only depend on $\Delta$. Then we define $M_{\Delta}:=<e_{0}, \pi^{n_{1}} e_{1}, \pi^{n_{2}} e_{2}>$. By construction $n_{1}+n_{2} \geq 0$. This gives a unique equivalence class $\left[M_{\Delta}\right]$ for $\Delta$. The stabilizer of $M_{\Delta}$ in $S U_{3}(L)$ is the group $P(\Delta)^{-}$. One easily sees that our simplicial structure $\mathscr{P}$ on the set of stable polyhedra corresponds with the simplicial structure of the modules $\left[M_{\Delta}\right]$ coming from $\mathscr{H}$.

So we can embed the set of stable polyhedra simplicially into the building $B_{3}$ of $S L_{3}(L)$. One now easily concludes that the affinoids $Y\left\{\rho_{1}, \rho_{2}, \sigma\right\}, \rho_{1} \neq \rho_{2}$, in the covering $\mathscr{C}$ of $Y^{s}$ that correspond to triangles in the picture (See 3.1) are exactly the same as the affinoids that go with the corresponding chamber in the $S L_{3}(L)$ building in the affinoid covering of $Y_{\mathscr{H}}$. Moreover the component of the reduction of $Y^{s}$ corresponding to the stable polyhedron $\Delta$ is the same as the component of the reduction of $Y_{\mathscr{H}}$ associated to $M_{\Delta}$, if $\Delta$ is not a vertex of type 0 in $B$. The components do differ if $\Delta$ is a vertex.

REMARK 8.12. The component of the reduction of $Y^{s}$ belonging to $\Delta$ is a $\mathbb{P}_{L}^{2}$ with some points blown up. The number of points blown up is as follows. If $L / K$ is unramified the number of points blown up is:

$$
\begin{array}{lll}
q^{2}\left(q^{2}-q+1\right) & \text { if } & \Delta \text { is a vertex. } \\
q^{4}+q+1 & \text { if } & \Delta \text { is not a vertex. }
\end{array}
$$

If $L / K$ is ramified then the numbers are as follows: 


$$
\begin{array}{llll}
q(q+1) / 2 & \text { if } \Delta & \text { is a vertex. } \\
q^{2}+q+1 & \text { if } \Delta \quad \text { is not a vertex and } v_{B}(\Delta) \quad \text { is a vertex of type } 1 . \\
q^{2}+2 & \text { if } \Delta \quad \text { is not a vertex and } v_{B}(\Delta) \quad \text { is a vertex of type } 0 .
\end{array}
$$

For the convenience of the reader we give in the table below the number of simplices $\left\{\Delta, \Delta^{\prime}\right\} \in \mathscr{P}$. We assume $|\Delta|=|\Delta \cap A|$. Each $P(\Delta)^{-}$orbit is determined by the

\begin{tabular}{|c|c|c|c|}
\hline$\Delta$ & $\left|\Delta^{\prime}\right|-|\Delta|$ & $L / K$ unramified & $L / K$ ramified \\
\hline$[2 i, 2 i]=[2 i]$ & $\begin{array}{l}2 \\
4\end{array}$ & $\begin{array}{c}q^{3}+1 \\
q^{2}\left(q^{2}-q+1\right)\end{array}$ & $\begin{array}{c}q+1 \\
q(q+1) / 2\end{array}$ \\
\hline$[2 i, 2 i+2]$ & $\begin{array}{c}-2 \\
2 \\
4\end{array}$ & $\begin{array}{c}q+1 \\
q^{2}(q+1) \\
q^{4}\end{array}$ & $\begin{array}{c}q+1 \\
q(q+1) \\
q^{2}\end{array}$ \\
\hline $\begin{array}{c}{[2 i, 2 j], \quad i<j} \\
i \neq j \quad \bmod 2 \\
j \neq i+1\end{array}$ & $\begin{array}{c}-4 \\
-2 \\
2 \\
4\end{array}$ & $\begin{array}{c}1 \\
q+1 \\
q^{2}(q+1) \\
q^{4}\end{array}$ & $\begin{array}{c}1 \\
q+1 \\
q(q+1) \\
q^{2}\end{array}$ \\
\hline $\begin{array}{l}{[2 i, 2 j], \quad i<j} \\
i=j \quad \bmod 2\end{array}$ & $\begin{array}{c}-4 \\
-2 \\
2 \\
4\end{array}$ & $\begin{array}{c}1 \\
q+1 \\
q^{2}(q+1) \\
q^{4}\end{array}$ & $\begin{array}{c}1 \\
2 \\
2 q \\
q^{2}\end{array}$ \\
\hline
\end{tabular}
difference $\left|\Delta^{\prime}\right|-|\Delta|$. We give the number of elements in the $P(\Delta)^{-}$orbit. They differ if $L / K$ is ramified or not.

8.13. The quotient $Y^{s} / \Gamma$. Let $\Gamma \subset S U_{3}(L)$ be a torsion-free discrete co-compact subgroup. The quotient $Y^{s} / \Gamma$ is a separated rigid analytic space. Since $\Gamma$ has infinitely many orbits on the components of the reduction of $Y^{s}$, the quotient is not proper. Moreover I would guess that the quotient itself cannot be compactified. To explain this, consider an easier example.

Let $F$ be the subgroup $S L_{2}$ of $S U_{3}$ that preserves the quadratic form $g(x)=$ $x_{1} x_{2}+x_{0}^{2}$. We also let $F$ act diagonally on $\mathbb{P}_{K^{0}}^{1} \times \mathbb{P}_{K^{0}}^{1}$. One has an $F$-equivariant map $\psi: \mathbb{P}^{1} \times \mathbb{P}^{1} \longrightarrow \mathbb{P}^{2}$ given by: $\psi(y, z)=\left(-\left(y_{1} z_{2}+y_{2} z_{1}\right), 2 y_{1} z_{1}, 2 y_{2} z_{2}\right)$. The map $\psi$ is $2: 1$ and ramifies along $g(x)=0$. We will also denote $\psi \otimes K$ by $\psi$.

Let $S(K) \subset F(K) \subset S U_{3}(L)$ be a maximal $K$-split torus. Let $A \subset B$ be the apartment belonging to $S$. We put $Y_{F}^{s}:=\bigcap_{g \in F(K)} g\left(Y_{A}^{s}\right)$ and $Y_{F}^{s s}:=\bigcap_{g \in F(K)} g\left(Y_{A}^{s s}\right)$. We also define $\tilde{Y}_{F}^{s s}:=Y_{F}^{s s}-\{g((1,0,0)) \mid g \in F(K)\}$, where $(1,0,0)$ is the point fixed by $S$. Let $\Omega_{1}:=\mathbb{P}^{1} \otimes K-\mathbb{P}^{1}(K)$ be Drinfeld's symmetric space. One easily proves the following: 
(1) $\psi^{-1}\left(Y_{F}^{s}\right)=\Omega_{1} \times \Omega_{1}$.

(2) $\psi^{-1}\left(\tilde{Y}_{F}^{s s}\right)=\left(\mathbb{P}_{K}^{1} \times \Omega_{1}\right) \cup\left(\Omega_{1} \times \mathbb{P}_{K}^{1}\right)$.

(3) $\psi\left(\mathbb{P}_{K}^{1} \times \Omega_{1}\right)=\tilde{Y}_{F}^{s s}$.

Let $\Gamma_{F} \subset F(K)$ be a discrete co-compact subgroup. Then $\left(\mathbb{P}_{K}^{1} \times \Omega_{1}\right) / \Gamma_{F}$ is a projective variety, whereas $Y_{F}^{s} / \Gamma_{F}$ is a separated non-proper variety. The restriction of the map $\psi$ to $\psi^{-1}\left(\tilde{Y}_{F}^{s s}\right)$ is a finite rigid analytic map. Since $\psi^{-1}\left(\tilde{Y}_{F}^{s s}\right) / \Gamma_{F}$ is not separated, the same is true of $\tilde{Y}_{F}^{s s} / \Gamma_{F}$. So it appears very difficult (impossible?) to compactify the quotient $Y_{F}^{s} / \Gamma_{F}$. However $\psi^{-1}\left(Y_{F}^{s}\right) / \Gamma_{F}$ is an open subspace of $\left(\mathbb{P}_{K}^{1} \times \Omega_{1}\right) / \Gamma_{F}$.

Let $I_{F}(x):=\left\{z \in B_{F} \mid \forall\left(A \subset B_{F} \wedge A \ni z\right) z \in I_{A}(x)\right\}$, where $B_{F}:=\bigcup_{g \in F(K)} g \cdot A \subset$ $B$ is the building of $F(K)$. Let $\mathscr{Z}_{F}:=\mathbb{P}_{K}^{1} \times \Omega_{1}-\left\{(y, z) \in \Omega_{1} \times \Omega_{1} \mid I(y)_{\mathbb{P}^{1}}=I(z)_{\mathbb{P}^{1}}\right\}$, where $I(-)_{\mathbb{P} 1}$ is as in 5.2. The map $(y, z) \longrightarrow\left(\psi(y, z), I(z)_{\mathbb{P}^{1}}\right)$ identifies $\mathscr{Z}_{F}$ with the set of pairs $(x, p)$ with $x \in \tilde{Y}_{F}^{s s}$ such that $\left|I_{F}(x)\right|>0$ and $p$ an extremal point of $I_{F}(x)$. So $\mathscr{Z}_{F}$ can be constructed from $\tilde{Y}^{s s}$.

The case of $Y^{s} / \Gamma$ seems to be similar. One takes $\tilde{Y}^{s s}:=Y^{s s}-\{g((1,0,0)) \mid g \in$ $\left.S U_{3}(L)\right\}$. One defines a space $\mathscr{Z}$ as consisting of the pairs $(x, p)$ with $x \in \tilde{Y}^{s s}$ and $I(x)$ not a point and $p$ an extremal point of $I(x)$. Now it is hoped that $\mathscr{Z} / \Gamma$ can be compactified ( instead of $Y^{s} / \Gamma$ ).

\section{References}

[1] S. Bosch, U. Güntzer and R. Remmert, Non-archimedean analysis (Springer, Berlin, 1984).

[2] J. Fresnel and M. van der Put, Geométrie analytique rigide et applications, Prog. Math. 18 (Birkhäuser, Boston, 1981).

[3] F. Bruhat and J. Tits, 'Groupes Réductifs sur un corps local I: Données radicielles valuées', Inst. Hautes Études Sci. Publ. Math. 41 (1972), 5-251.

[4] M. van der Put and H. Voskuil, 'Symmetric spaces associated to split algebraic groups over a local field', J. Reine Angew. Math. 433 (1992), 69-100.

[5] G. A. Mustafin, 'Nonarchimedean uniformization', Math. USSR-Sb. 34 (1978), 187-214.

[6] T. Oda, Convex bodies and algebraic geometry (Springer, Berlin, 1988).

[7] J. Tits, 'Reductive groups over local fields', Proc. Amer. Math. Soc. Symp. Pure Math. 33 (1979), 29-69.

[8] H. Voskuil, Non-archimedean Hopf Surfaces, Séminaire de Théorie des Nombres de Bordeaux 3(1991), 405-466.

[9] H. Voskuil, 'P-adic symmetric spaces: The unitary group acting on the projective plane', in: Algebraic geometry symposium at Kinosaki, 1993 (ed. prof. Maruyama) (Kyoto University Press, Kyoto, 1994), pp. 58-76.

School of Mathematics and Statistics

University of Sydney

NSW 2006

Australia

e-mail: voskuil_h@maths.su.oz.au 